Pacific

Journal of

Mathematics

CONFORMAL DESIGNS AND

MINIMAL CONFORMAL WEIGHT SPACES

OF VERTEX OPERATOR SUPERALGEBRAS

TOMONORI HASHIKAWA 


\title{
CONFORMAL DESIGNS AND MINIMAL CONFORMAL WEIGHT SPACES OF VERTEX OPERATOR SUPERALGEBRAS
}

\author{
TOMONORI HASHIKAWA
}

\begin{abstract}
We give equivalent conditions for conformal designs of the minimal conformal weight spaces of SVOAs, and show that if the minimal conformal weight space of an SVOA forms a conformal $2 \boldsymbol{m}$-design, then it also forms a conformal $(2 m+1)$-design. Also, we derive trace formulae for the zero-modes of elements of the conformal weight 2 space on the minimal conformal weight space when the minimal conformal weight space forms a conformal 4-design. As an application of the trace formulae, we classify code SVOAs whose minimal conformal weight spaces form conformal 4-designs. Moreover, we show that the classified code SVOAs are of class $\mathcal{S}^{5}$.
\end{abstract}

\section{Introduction}

Vertex operator algebras (VOA) and vertex operator superalgebras (SVOA) have deep connections to binary codes, integral lattices, and other combinatorial objects. The notion of conformal designs was introduced in [Höhn 2008], and is an analogue of the notions of combinatorial and spherical designs based on binary codes and integral lattices, respectively. Also, an analogue of the theorems of Assmus and Mattson [1969] and Venkov [2001] was presented in the same work. Due to this result, we expect that analogues of other properties of combinatorial and spherical designs hold in the theory of conformal designs. An integral lattice whose set of minimum norm vectors is a spherical design has been studied in [loc. cit.]. As one of the results, it was proved that an integral lattice whose set of minimum norm vectors forms a spherical 4-design, which is called a strongly perfect lattice, is isomorphic to the root lattices $A_{1}, A_{2}, D_{4}, E_{6}, E_{7}$, or $E_{8}$ if its minimum norm is 2 . Also, strongly perfect lattices with minimum norm 3 have been classified in the same paper. Due to these circumstances, we speculate that a structural symmetry of an algebraic object is dominated by a subset which has a design structure. From this point of view, our purpose of this study is to clarify how the symmetry of the minimal conformal weight space of an SVOA influences a structural symmetry of

MSC2010: 17B69.

Keywords: vertex operator superalgebra, conformal design, binary code. 
the SVOA. VOAs of class $\mathcal{S}^{n}$ with minimal conformal weight 2 have been discussed in [Matsuo 2001]. The notion of VOAs of class $\mathcal{S}^{n}$ was introduced in the same paper, and gives a sufficient condition that the minimal conformal spaces of VOAs form conformal $n$-designs. The conformal designs have been studied in [Yamauchi 2014] under the assumption for introducing the notion of extended Griess algebras. Considering these known results, we maintain the theory of the minimal conformal weight spaces of SVOAs and conformal designs. The following are the main results obtained in Section 3 of this paper.

Main Result 1 (Theorems 3.5 and 3.6). Let $V$ be an SVOA and $\mu$ the minimal conformal weight of $V$. Assume that $\mu<\infty$. Then the following hold:

(1) The space $V_{\mu}$ forms a conformal $t$-design based on the even part of $V$ if and only if the $t$-th Casimir vector, introduced in [Matsuo 2001], belongs to the sub-VOA $V_{\omega}$ generated by the Virasoro element $\omega$.

(2) If $V_{\mu}$ is a conformal $2 m$-design based on the even part of $V$, then it is also a conformal $(2 m+1)$-design.

By using the computation of traces and invariant bilinear forms in [Yamauchi 2014], we have (1) of Main Result 1. The crucial point of the proof of (2) of Main Result 1 is that the $(2 m+1)$-th Casimir vector can be determined from the $n$-th Casimir vectors for $n \leq 2 m$ and the action of $L(-1)$. Note that (2) is an analogue of a well-known result in the theory of integral lattices and spherical designs. Moreover, trace formulae of the zero-modes of elements of the conformal weight-2 space on the minimal conformal weight space of an SVOA are obtained when the minimal conformal weight space forms a conformal 4-design.

As another related topic of conformal designs and SVOAs, there are classification problems of SVOAs whose minimal conformal weight spaces form conformal $t$-designs. This problem has been solved in [Höhn 2008] for the case that the minimal conformal weight is 1 and $t=6$. More precisely, SVOAs with minimal conformal weight 1 are isomorphic to lattice VOAs associated to the root lattices of type $A_{1}$ and $E_{8}$ if the conformal weight-1 space forms a conformal 6-design. Also, it was proved in [Tuite 2009] that a VOA whose 4 th Casimir vector belongs to $V_{\omega}$ is isomorphic to one of the simple affine VOA associated to the Deligne exceptional series of the simple Lie algebras $A_{1}, A_{2}, G_{2}, D_{4}, F_{4}, E_{6}, E_{7}$, and $E_{8}$ at level 1 if the minimal conformal weight is 1 . Using (1) of Main Result 1, this classification result can be obtained under the condition that the conformal weight- 1 space forms a conformal 4-design. This result is actually an analogue of the result in [Venkov 2001], as already mentioned. Due to the classification in [Tuite 2009], one can consider the classification problem in the case of SVOAs with minimal conformal weight $\frac{3}{2}$ and $t=4$. The commutant superalgebra (see [Yamauchi 2005]) of an Ising vector in the lattice type VOA $V_{\sqrt{2}}^{+} E_{8}$ is included in the list of candidates of 
SVOAs with minimal conformal weight $\frac{3}{2}$ whose 4 th Casimir vector belongs to $V_{\omega}$ (see [Tuite and Van 2014]), and is isomorphic to the code SVOA $V_{\mathcal{H}_{4}}$ associated to the Hamming code $\mathcal{H}_{4}$ (see [Miyamoto 1996a] for the definition of code SVOAs). The notion of SVOAs of class $\mathcal{S}^{n}$ is an analogue of the ordinary notion introduced in [Matsuo 2001] and gives a sufficient condition that the minimal conformal weight spaces of SVOAs form conformal $n$-designs in the same way as the cases of VOAs of class $\mathcal{S}^{n}$.

In this paper, we show that $V_{\mathcal{H}_{4}}$ is of class $\mathcal{S}^{5}$. Moreover, we classify code SVOAs whose minimal conformal weight spaces form conformal 4-designs as an application of the results in Section 3 and show that the classified code SVOAs, which contain $V_{\mathcal{H}_{4}}$, are of class $\mathcal{S}^{5}$. We obtain the following.

Main Result 2 (Theorems 4.8 and 5.9). Let $C$ be a binary code. Assume that the minimal conformal weight $\mu$ of the code $S V O A V_{C}$ is not $\infty$. Then:

(1) If $\left(V_{C}\right)_{\mu}$ forms a conformal 4-design based on the even part, then $C$ is equivalent to one of

$$
\left\{\left(0^{1}\right),\left(1^{1}\right)\right\}, \hat{\mathcal{H}}_{3}, \mathcal{E}_{8}, E\left(\mathcal{H}_{4}\right), \mathcal{H}_{4} \text {, and } \hat{\mathcal{H}}_{4},
$$

where $\mathcal{H}_{m}, E\left(\mathcal{H}_{m}\right), \hat{\mathcal{H}}_{m}$, and $\mathcal{E}_{8}$ are the Hamming code of length $2^{m}-1$, the even subcode of $\mathcal{H}_{m}$, the extended Hamming code of $\mathcal{H}_{m}$, and the set of all even weight vectors in $\mathbb{F}_{2}^{8}$, respectively.

(2) The code SVOAs associated to the codes in (1) are of class $\mathcal{S}^{5}$.

We see that for a code SVOA the minimal conformal weight space forms a conformal 4-design if and only if the SVOA is of class $\mathcal{S}^{5}$.

In the following, we sketch the proof of Main Result 2. Let $C$ be a binary code of length $n$ and $\mu$ the minimal conformal weight of $V_{C}$. Obviously, we can exclude the case $\mu>2$. Considering the trace formulae on the minimal conformal weight space, $n=1,8$, and 15 if $\mu=\frac{1}{2}, 1$, and $\frac{3}{2}$, respectively. In case $\mu=\frac{1}{2}, C$ must be $\left\{\left(0^{1}\right),\left(1^{1}\right)\right\}$ because it has a weight- 1 vector. In cases $\mu=1, \frac{3}{2}$, and 2 , we show that $\left(\Omega_{n}, C(2 \mu)\right)$ is a combinatorial 2-design if $\left(V_{C}\right)_{\mu}$ is a conformal 4-design, where $\Omega_{n}:=\{1, \ldots, n\}$ and $C(2 \mu)$ is the set of all weight $2 \mu$ vectors in $C$. By this result, $C \cong \mathcal{E}_{8}$ if $\mu=1$, and $C \cong \mathcal{H}_{4}$ if $\mu=\frac{3}{2}$. Also, we have $C \cong \widehat{\mathcal{H}}_{3}, E\left(\mathcal{H}_{4}\right)$, or $\hat{\mathcal{H}}_{4}$ by using fundamental techniques of algebraic coding theory and a list of possible central charges of VOAs with $\mu=2$ which is obtained in [Matsuo 2001]. Thus (1) of Main Result 2 holds. Now we turn to (2) of that result. Obviously,

$$
V_{\left\{\left(0^{1}\right),\left(1^{1}\right)\right\}}=L\left(\frac{1}{2}, 0\right) \oplus L\left(\frac{1}{2}, \frac{1}{2}\right)
$$

is of class $\mathcal{S}^{\infty}$. Note that the code SVOAs $V_{\mathcal{E}_{8}}, V_{\widehat{\mathcal{H}}_{3}}$, and $V_{\widehat{\mathcal{H}}_{4}}$ have already been proved; see [Maruoka et al. 2016; Hashikawa and Shimakura 2016]). Also, if $V_{\mathcal{H}_{4}}$ is of class $\mathcal{S}^{5}$, then so is $V_{E\left(\mathcal{H}_{4}\right)}$ because $V_{E\left(\mathcal{H}_{4}\right)}$ is the even part of $V_{\mathcal{H}_{4}}$. Hence 
it is sufficient to show that $V_{\mathcal{H}_{4}}$ is of class $\mathcal{S}^{5}$. Using the same method as in [Lam et al. 2007, Propositions 3.13], one can show that the automorphism group of a code SVOA is generated by $\sigma$-involutions and the lift of the automorphism group of the binary code if the minimum weight of the code is greater than or equal to 3 . Considering the action of a $\sigma$-involution associated to an Ising vector of $\sigma$-type which is not included in the standard Ising frame of $V_{\mathcal{H}_{4}}$, we prove that $V_{\mathcal{H}_{4}}$ is of class $\mathcal{S}^{5}$. Therefore, (2) holds.

This paper is organized as follows. In Section 2, we recall the notions of SVOAs and Ising vectors of SVOAs. In Section 3, we recall the notions of conformal designs, give necessary and sufficient conditions for conformal designs of minimal conformal weight spaces, and show that conformal $2 m$-designs imply conformal $(2 m+1)$ designs. Also, we give trace formulae on the minimal conformal weight space of an SVOA by using the same argument as in [Matsuo 2001]. In Section 4, using the trace formulae obtained in Section 3, we classify code SVOAs whose minimal conformal weight spaces form conformal 4-designs. In Section 5, we show that the code SVOAs associated to the codes in the classification of Section 4 are of class $\mathcal{S}^{5}$.

\section{Preliminaries}

In this section, we recall the notion of vertex operator superalgebras and Ising vectors. Additionally, we show an analogue of [Höhn et al. 2012, Lemma 2.6], which will be used in Section 3.

Vertex operator superalgebras. A vertex operator superalgebra (SVOA)

$$
V=V^{0} \oplus V^{1}
$$

is a $\mathbb{Z}_{2}$-graded $\mathbb{C}$-vector space equipped with a linear map

$$
Y(\cdot, z): V \rightarrow \operatorname{End}(V) \llbracket z, z^{-1} \rrbracket, \quad v \mapsto \sum_{n \in \mathbb{Z}} v_{(n)} z^{-n-1}
$$

and two nonzero vectors 1 and $\omega$ in $V^{0}$, which are called the vacuum vector and the Virasoro element, respectively, satisfying certain conditions; see [Frenkel et al. 1993; Kac 1998] for details. As one of the conditions, the Virasoro relation holds on $V$ :

$$
[L(m), L(n)]=(m-n) L(m+n)+\frac{m^{3}-m}{12} \delta_{m+n, 0} c
$$

for $m, n \in \mathbb{Z}$, where $Y(\omega, z)=\sum_{n \in \mathbb{Z}} L(n) z^{-n-2}, \delta_{i j}$ is the Kronecker symbol, and $c \in \mathbb{C}$ is the central charge of $V$. The subspaces $V^{0}$ and $V^{1}$ are called the even part and the odd part of $V$, respectively. Throughout the paper, we assume that an SVOA $V$ has the following grading:

$$
V^{0}=\bigoplus_{n \in \mathbb{Z}_{\geq 0}} V_{n} \quad \text { and } \quad V^{1}=\bigoplus_{n \in \frac{1}{2}+\mathbb{Z}_{\geq 0}} V_{n},
$$


where $V_{n}$ is the eigenspace of the $L(0)$-operator with eigenvalue $n$. We also assume that $V$ is of CFT-type, i.e., $V_{0}=\mathbb{C} 1$. An SVOA $V=V^{0} \oplus V^{1}$ is said to be a vertex operator algebra (VOA) if $V^{1}=0$. If $u \in V_{m}$, then we write $\operatorname{wt}(u):=m$. Define the zero-mode of a homogeneous element $u$ by $o(u):=u_{(\mathrm{wt}(u)-1)}$, and extend linearly.

Let $V_{\omega}$ denote the sub-VOA of an SVOA $V$ generated by the Virasoro element $\omega$. Then the minimal conformal weight of $V$ is defined by $\min \left\{n \in \frac{1}{2} \mathbb{Z}_{\geq 0} \mid V_{n} \neq\left(V_{\omega}\right)_{n}\right\}$ if $V \neq V_{\omega}$ and $\infty$ if $V=V_{\omega}$. Since we assume that $V$ is of CFT-type, the minimal conformal weight of $V$ is always greater than zero throughout this paper.

An element $\sigma$ of $\mathrm{GL}(V)$ is called an automorphism of an SVOA $V$ if it satisfies

$$
\sigma\left(u_{(m)} v\right)=\sigma(u)_{(m)} \sigma(v) \text { for all } u, v \in V, m \in \mathbb{Z} \text {, and } \sigma(\omega)=\omega .
$$

Let $\operatorname{Aut}(V)$ denote the group of all automorphisms of $V$.

Ising vectors of SVOAs. Let $V$ be an SVOA. An element $e \in V_{2}$ is called an Ising vector of $V$ if it satisfies $e_{(1)} e=2 e, e_{(3)} e=\frac{1}{4} 1$, and the subalgebra $\operatorname{Vir}(e)$ generated by $e$ is isomorphic to the simple Virasoro VOA $L\left(\frac{1}{2}, 0\right)$ with central charge $\frac{1}{2}$. It is known that $L\left(\frac{1}{2}, 0\right)$ is rational and has three irreducible modules

$$
L\left(\frac{1}{2}, 0\right), \quad L\left(\frac{1}{2}, \frac{1}{2}\right), \quad \text { and } \quad L\left(\frac{1}{2}, \frac{1}{16}\right) ;
$$

see [Dong et al. 1994, Theorem 3.4] for details. Let $e$ be an Ising vector of $V$. Note that $\left\{L^{e}(n):=e_{(n+1)} \mid n \in \mathbb{Z}\right\}$ satisfies the Virasoro relation with central charge $\frac{1}{2}$. Since $\operatorname{Vir}(e) \cong L\left(\frac{1}{2}, 0\right)$, we have a decomposition

$$
V=V_{e}(0) \oplus V_{e}\left(\frac{1}{2}\right) \oplus V_{e}\left(\frac{1}{16}\right),
$$

where $V_{e}(k)$ for $k \in\left\{0, \frac{1}{2}, \frac{1}{16}\right\}$, is the sum of all irreducible $\operatorname{Vir}(e)$-submodules of $V$ isomorphic to $L\left(\frac{1}{2}, k\right)$. Let $\mu$ be the minimal conformal weight of $V$. Set

$$
W_{\mu}^{e}(k):=\left\{u \in V_{\mu} \mid o(e) u=k u\right\} .
$$

The following lemma is an analogue of [Höhn et al. 2012, Lemma 2.6].

Lemma 2.1. Let $V$ be an $S V O A$ and e an Ising vector. If $\mu \in\{1\} \cup\left(\frac{1}{2}+\mathbb{Z}_{\geq 0}\right)$, then

$$
V_{\mu}=W_{\mu}^{e}(0) \oplus W_{\mu}^{e}\left(\frac{1}{2}\right) \oplus W_{\mu}^{e}\left(\frac{1}{16}\right) .
$$

Proof. Since $o(e)$ preserves $V_{\mu}$ and acts semisimply on $V$, the space can be decomposed into the direct sum of the eigenspaces of $o(e)$. Let $v \in V_{\mu}$ be an eigenvector of $o(e)$ with eigenvalue $\lambda$. It is sufficient to show that $\lambda \in\left\{0, \frac{1}{2}, \frac{1}{16}\right\}$. In case $\mu \in \frac{1}{2}+\mathbb{Z}_{\geq 0}$ we have $L^{e}(m) v \in V_{\mu-m}=0$ for $m \geq 1$, and hence $\operatorname{Vir}(e) v$ is a $\operatorname{Vir}(e)$-module whose top weight is $\lambda$. Since $\operatorname{Vir}(e) \cong L\left(\frac{1}{2}, 0\right)$, this case holds. For $\mu=1$, we have $L^{e}(m) v \in V_{1-m}=0$ for $m \geq 2$. If we suppose that $L^{e}(1) v=0$, then this case also holds by using the same method as in the case of $\mu \in \frac{1}{2}+\mathbb{Z}_{\geq 0}$. We show $L^{e}(1) v=0$. Suppose the claim is not true. Since $o(e) L^{e}(1) v=(\lambda-1) L^{e}(1) v$ 
and $o(e) \mathbf{1}=e_{(1)} \mathbf{1}=0$, we have $\lambda=1$. However, this contradicts the nonexistence of an $o(e)$-weight-1 vector.

Here, we give the definitions of Ising vectors of $\sigma$-type and Ising frames, which will be used later. An Ising vector $e$ of an SVOA $V$ is said to be of $\sigma$-type if $V_{e}\left(\frac{1}{16}\right)=0$. For an Ising vector $e$ of $\sigma$-type, the linear map

$$
\sigma_{e}:=\left\{\begin{aligned}
1 & \text { on } V_{e}(0) \\
-1 & \text { on } V_{e}\left(\frac{1}{2}\right),
\end{aligned}\right.
$$

is an automorphism of $V$; see [Miyamoto 1996b, Theorem 4.8]. A subset $\left\{e^{1}, \ldots, e^{n}\right\}$ of $V_{2}$ such that $\omega=e^{1}+\cdots+e^{n}$ is called an Ising frame if $e^{i}$ is an Ising vector of $V$ for each $1 \leq i \leq n$ and $\left[Y\left(e^{i}, z\right), Y\left(e^{j}, z\right)\right]=0$ for $i \neq j$.

\section{Conformal designs}

In this section, we first review the notion of conformal designs, and obtain necessary and sufficient conditions in the case where the minimal conformal weight spaces of SVOAs form conformal designs. Also, we show that if the minimal conformal weight space of an SVOA forms a conformal $2 m$-design, then it also forms a conformal $(2 m+1)$-design. Afterward, we give trace formulae of the composition of the zero-modes of elements of $V_{2}$ on the minimal conformal weight space when the space forms a conformal 4-design.

Conditions of SVOAs. Set $\zeta^{r}=e^{\pi \sqrt{-1} r}$ for $r \in \mathbb{Q}$, and let $V$ be an SVOA. A bilinear form $(\cdot \mid \cdot)$ on $V$ is said to be invariant if it satisfies

$$
(Y(a, z) u \mid v)=\left(u \mid Y\left(e^{z L(1)} z^{-2 L(0)} \zeta^{L(0)+2 L(0)^{2}} a,-z\right) v\right)
$$

for $a, u, v \in V$. It was proved in [Frenkel et al. 1993; Li 1994; Yamauchi 2014] that any invariant bilinear form on an SVOA is symmetric and there is a one-to-one correspondence between invariant bilinear forms and elements of the dual space of $V_{0} / L(1) V_{1}$. In this paper, we assume that $V$ has a nondegenerate invariant bilinear form $(\cdot \mid \cdot)$. Due to the results above, the bilinear form is unique up to scalar since $V$ is of CFT-type. Moreover, we assume that $V$ as a $V_{\omega}$-module is a direct sum of highest weight modules. Hence

$$
V=\bigoplus_{n \in \frac{1}{2} \mathbb{Z} \geq 0} V[n],
$$

where $V[n]$ is the sum of highest weight $V_{\omega}$-submodules of $V$ with highest weight $n \in \frac{1}{2} \mathbb{Z}$. Note that $V[0]=V_{\omega}$ holds. We have the following lemma.

Lemma 3.1. The spaces $V[0]$ and $V[m]$ for $m \neq 0$ are orthogonal with respect to $(\cdot \mid \cdot)$. 
Proof. Let $n \in \mathbb{Z}_{\geq 0}, u \in V[0] \cap V_{n}$, and $v \in V[m] \cap V_{n}$. By the invariance of $(\cdot \mid \cdot)$,

$$
(u \mid v)=\sum_{\ell \geq 0} \frac{\zeta^{n+2 n^{2}}}{\ell !}\left(\mathbf{1} \mid\left(L(1)^{\ell} u\right)_{(2 n-1-\ell)} v\right) .
$$

Since $V[m]$ is a $V_{\omega}$-module, $\left(L(1)^{\ell} u\right)_{(2 n-1-\ell)} v$ belongs to $V[m] \cap V_{0}$ for each $\ell \geq 0$. Because $V_{0} \subset V[0]$ and $V[0] \cap V[m]=0$, the right hand side of (3-1) is 0 . Therefore, we have this lemma because $\left(V_{k} \mid V_{k^{\prime}}\right)=0$ for $k \neq k^{\prime}$.

Define the projection map

$$
\pi: V=\bigoplus_{n \in \frac{1}{2} \mathbb{Z} \geq 0} V[n] \rightarrow V[0]=V_{\omega}
$$

which is a $V_{\omega}$-module homomorphism.

Minimal conformal weight spaces and conformal designs. The notion of conformal designs was introduced by Höhn in [2008].

Definition 3.2 [Höhn 2008, Section 2]. Let $U$ be a VOA and $M$ a $U$-module. An $L(0)$-homogeneous subspace $X$ of $M$ is called a conformal $t$-design based on $U$ if $\left.\operatorname{tr}\right|_{X} o(a)=\left.\operatorname{tr}\right|_{X} o(\pi(a))$ holds for any $a \in \bigoplus_{0 \leq n \leq t} U_{n}$.

Let $V=V^{0} \oplus V^{1}$ be an SVOA. Clearly, $V^{0}$ and $V^{1}$ are $V^{0}$-modules. From now on, we assume that the minimal conformal weight $\mu$ of $V$ is not $\infty$.

Remark 3.3. Assume that $V$ has an involution $g$. Set $V^{ \pm}:=\{u \in V \mid g(u)= \pm u\}$. Yamauchi [2014] considered that the top weight space of $V^{-}$forms a conformal design based on $V^{0} \cap V^{+}$under some assumptions, and obtained various results. However, these results do not contain the general cases $\mu \in\left\{\frac{1}{2}, 1\right\}$. We are going to include these general cases in our discussion.

By Lemma 3.1, $(\cdot \mid \cdot)$ is also nondegenerate on $\left(V_{\omega}\right)_{\mu}$ and $P_{\mu}$, where

$$
P_{\mu}:=\left\{u \in V_{\mu} \mid L(k) u=0 \text { for all } k \in \mathbb{Z}_{\geq 0}\right\} .
$$

Moreover, $V_{\mu}=\left(V_{\omega}\right)_{\mu} \oplus P_{\mu}$ holds because

$$
V=\bigoplus_{n \in \frac{1}{2} \mathbb{Z} \geq 0} V[n]
$$

Let $\left\{v_{i}\right\}_{i=1}^{p_{\mu}}$ be a basis of $P_{\mu}$, and $\left\{v^{i}\right\}_{i=1}^{p_{\mu}}$ the dual basis of $\left\{v_{i}\right\}_{i=1}^{p_{\mu}}$ with respect to $(\cdot \mid \cdot)$, where $p_{\mu}:=\operatorname{dim} P_{\mu}$. We consider the vector

$$
\lambda_{\mu}^{m}:=\zeta^{\mu+2 \mu^{2}} \sum_{i=1}^{p_{\mu}} v_{(2 \mu-1-m)}^{i} v_{i} \in V_{m} .
$$


Note that $\zeta^{\mu+2 \mu^{2}} \lambda_{\mu}^{m}$ is called the quadratic Casimir vector in [Tuite 2009; Tuite and Van 2014]. We also note that if $\lambda_{\mu}^{m} \in V_{\omega}$, then $\lambda_{\mu}^{\ell} \in V_{\omega}$ for $\ell \leq m$; see [loc. cit.]. Set $q_{\mu}:=\operatorname{dim}\left(V_{\omega}\right)_{\mu}$. Let $\left\{w_{i}\right\}_{i=1}^{q_{\mu}}$ be a basis of $\left(V_{\omega}\right)_{\mu}$ and $\left\{w^{i}\right\}_{i=1}^{q_{\mu}}$ the dual basis of $\left\{w_{i}\right\}_{i=1}^{q_{\mu}}$ with respect to $(\cdot \mid \cdot)$. The following lemma holds.

Lemma 3.4. Let $V$ be an SVOA with minimal conformal weight $\mu$. Then

$$
\left.\operatorname{tr}\right|_{V_{\mu}} o(u)=(-1)^{\mathrm{wt}(u)}\left(u \mid \lambda_{\mu}^{\mathrm{wt}(u)}\right)
$$

for a homogeneous element $u \in \bigoplus_{n \in \mathbb{Z}_{>0}} V[n]$.

Proof. Because $\left\{v^{i}\right\}_{i=1}^{p_{\mu}} \cup\left\{w^{i}\right\}_{i=1}^{q_{\mu}}$ is the dual basis of $\left\{v_{i}\right\}_{i=1}^{p_{\mu}} \cup\left\{w_{i}\right\}_{i=1}^{q_{\mu}}$ with respect to $(\cdot \mid \cdot)$,

$$
\left.\operatorname{tr}\right|_{V_{\mu}} o(u)=\sum_{i=1}^{p_{\mu}}\left(o(u) v^{i} \mid v_{i}\right)+\sum_{i=1}^{q_{\mu}}\left(o(u) w^{i} \mid w_{i}\right) .
$$

Since $o(u) w^{i} \in \bigoplus_{n>0} V[n]$ and $w_{i} \in V[0]$, the second summation of (3-2) is 0 by Lemma 3.1, i.e., $\left.\operatorname{tr}\right|_{V_{\mu}} o(u)=\sum_{i=1}^{p_{\mu}}\left(o(u) v^{i} \mid v_{i}\right)$ holds. By the same computation as in [Yamauchi 2014, Section 4.1, Lemma 5], we obtain the statement.

Set $d_{\mu}:=\operatorname{dim} V_{\mu}$, let $\left\{u_{i}\right\}_{i=1}^{d_{\mu}}$ be a basis of $V_{\mu}$, and let $\left\{u^{i}\right\}_{i=1}^{d_{\mu}}$ be its dual basis with respect to $(\cdot \mid \cdot)$ ). We also consider the following Casimir vector (see [Matsuo 2001; Yamauchi 2014]):

$$
\kappa_{\mu}^{m}:=\zeta^{\mu+2 \mu^{2}} \sum_{i=1}^{d_{\mu}} u_{(2 \mu-1-m)}^{i} u_{i}=\lambda_{\mu}^{m}+\zeta^{\mu+2 \mu^{2}} \sum_{i=1}^{q_{\mu}} w_{(2 \mu-1-m)}^{i} w_{i} \in V_{m} .
$$

We obtain the following equivalent conditions to define conformal designs. It has already been discussed in [Yamauchi 2014] for $\mu \in \frac{1}{2}+\mathbb{Z}_{\geq 1}$.

Theorem 3.5. Let $V$ be an SVOA with minimal conformal weight $\mu$. Then the following are equivalent: (1) $V_{\mu}$ is a conformal $t$-design based on $V^{0}$, (2) $\kappa_{\mu}^{t} \in V_{\omega}$, and (3) $\lambda_{\mu}^{t} \in V_{\omega}$.

Proof. By (3-3), (2) $\Longleftrightarrow$ (3) holds. We show (1) $\Longleftrightarrow$ (3). Let $a \in V_{t}^{0}$. Set $\bar{a}:=a-\pi(a)$. Then $\left.\operatorname{tr}\right|_{V_{\mu}} o(a)=\left.\operatorname{tr}\right|_{V_{\mu}} o(\pi(a))+(-1)^{t}\left(\bar{a} \mid \lambda_{\mu}^{t}\right)$ by Lemma 3.4. Therefore, $\left.\operatorname{tr}\right|_{V_{\mu}} o(a)=\left.\operatorname{tr}\right|_{V_{\mu}} o(\pi(a))$ if and only if $\left(\bar{a} \mid \lambda_{\mu}^{t}\right)=0$. We see from Lemma 3.1 that $\left(\bar{a} \mid \lambda_{\mu}^{t}\right)=0$ for any $a \in V_{t}^{0}$ if and only if $\lambda_{\mu}^{t} \in V[0]=V_{\omega}$.

It is known that if the set of minimum norm vectors of an integral lattice forms a spherical $2 m$-design, then it also forms $(2 m+1)$-design; see [Venkov 2001, Section 5, p. 23]. The assertion of the following theorem is an analogy of this particular result in the case of a conformal design. A method to prove this when $\mu=2$ was mentioned briefly in [Matsuo 2001, Section 2, p. 573]. 
Theorem 3.6. Let $V$ be an SVOA with minimal conformal weight $\mu$. If $V_{\mu}$ forms a conformal $2 m$-design based on the even part, then it also forms a conformal $(2 m+1)$-design.

Proof. By the skew symmetry,

$$
\begin{aligned}
& \kappa_{\mu}^{2 m+1}= \\
& \quad \sum_{\ell \geq 0} \frac{(-1)^{2 \mu-(2 m+1)+\ell}}{\ell !} L(-1)^{\ell}\left(\zeta^{\mu+2 \mu^{2}} \sum_{i=1}^{d_{\mu}}(-1)^{\left|u^{i}\right|\left|u_{i}\right|}\left(u_{i}\right)_{(2 \mu-1-(2 m+1)+\ell)} u^{i}\right),
\end{aligned}
$$

where $|a|$ equals 0 if $a \in V^{0}$, and 1 if $a \in V^{1}$. Since $\left|u^{i}\right|\left|u_{i}\right|=2 \mu \bmod 2$, we have

$$
\kappa_{\mu}^{2 m+1}=\sum_{\ell \geq 0} \frac{(-1)^{1+\ell}}{\ell !} L(-1)^{\ell} \kappa_{\mu}^{2 m+1-\ell} .
$$

Hence,

$$
\kappa_{\mu}^{2 m+1}=\frac{1}{2} \sum_{\ell \geq 1} \frac{(-1)^{1+\ell}}{\ell !} L(-1)^{\ell} \kappa_{\mu}^{2 m+1-\ell} .
$$

If $V_{\mu}$ forms a conformal $2 m$-design based on the even part, then by Theorem 3.5 $\kappa_{\mu}^{s} \in V_{\omega}$ for $1 \leq s \leq 2 m$. Therefore, using (3-4) and Theorem 3.5, we are done.

From now on, we assume that the central charge of an SVOA is neither 0 nor $-\frac{22}{5}$. This assumption implies that the degree $m$ subspace of $V_{\omega}$ with $m \leq 5$ has a basis

$$
\left\{L\left(-n_{1}\right) \cdots L\left(-n_{r}\right) \mathbf{1} \mid n_{1} \geq \cdots \geq n_{r} \geq 2, \sum_{i=1}^{r} n_{i}=m\right\} ;
$$

see [Kac and Raina 1987, Lecture 8].

We also assume that the bilinear form is normalized by $(\mathbf{1} \mid \mathbf{1})=1$. The following lemma holds.

Lemma 3.7. Let $V$ be an $S V O A, a, b \in V_{2}, m \in \mathbb{Z}_{\geq 2}$, and $n \in \mathbb{Z}$. Then:

(1) $\left(L(-m) \mathbf{1} \mid a_{(n)} b\right)=(2 m-2) \delta_{m+n, 3}(a \mid b)-\frac{m^{2}-3 m+4}{2} \delta_{m+n, 3}(L(1) a \mid L(1) b)$,

(2) $\left(L(-2)^{2} \mathbf{1} \mid a_{(-1)} b\right)=2(a \mid \omega)(b \mid \omega)+8(a \mid b)-4(L(1) a \mid L(1) b)$.

Proof. By the commutator formula, for $k, \ell \in \mathbb{Z}$,

$$
\begin{aligned}
& {\left[L(k), a_{(\ell)}\right]} \\
& \qquad=(k-\ell+1) a_{(k+\ell)}+\left(\begin{array}{c}
k+1 \\
2
\end{array}\right)(L(1) a)_{(k+\ell-1)}+\left(\begin{array}{c}
k+1 \\
3
\end{array}\right) \delta_{k+\ell, 1}(a \mid \omega) .
\end{aligned}
$$

Since $V$ is of CFT-type and has a nondegenerate invariant bilinear form, $L(1) V_{1}=0$. We compute $X:=\left(L(-m) \mathbf{1} \mid a_{(n)} b\right)$ :

$$
X=\underbrace{\left(\mathbf{1} \mid a_{(n)} L(m) b\right)}_{=0}+\left(\mathbf{1} \mid\left[L(m), a_{(n)}\right] b\right)
$$




$$
\begin{aligned}
& =(m-n+1)\left(\mathbf{1} \mid a_{(m+n)} b\right)+\left(\begin{array}{c}
m+1 \\
2
\end{array}\right)\left(\mathbf{1} \mid(L(1) a)_{(m+n-1)} b\right) \quad(\text { by }(3-5)) \\
& =\delta_{m+n, 3}\left((2 m-2)\left(\mathbf{1} \mid a_{(3)} b\right)+\left(\begin{array}{c}
m+1 \\
2
\end{array}\right)\left(\mathbf{1} \mid(L(1) a)_{(2)} b\right)\right) \\
& \quad\left(\text { since }\left(V_{k} \mid V_{\ell}\right)=0 \text { if } k \neq \ell\right) \\
& =\delta_{m+n, 3}\left((2 m-2)(a \mid b)-\frac{m^{2}-3 m+4}{2}\left((L(1) a)_{(-2)} \mathbf{1} \mid b\right)\right) \\
& \left.\quad \text { by invariance, } L(1) V_{1}=0\right) \\
& =\delta_{m+n, 3}\left((2 m-2)(a \mid b)-\frac{m^{2}-3 m+4}{2}(L(1) a \mid L(1) b)\right) . \\
& \quad\left(\text { since }(L(1) a)_{(-2)} \mathbf{1}=L(-1) L(1) a\right)
\end{aligned}
$$

Hence, we obtain (1). Next, we show (2). By $(1)$ for $(m, n)=(2,1)$, the invariance, and (3-5),

$$
\begin{aligned}
& \left(L(-2)^{2} \mathbf{1} \mid a_{(-1)} b\right) \\
& \quad=2(a \mid \omega)(b \mid \omega)+8(a \mid b)-4(L(1) a \mid L(1) b)+3\left(\omega \mid(L(1) a)_{(0)} b\right) .
\end{aligned}
$$

We show $\left(\omega \mid(L(1) a)_{(0)} b\right)=0$. By (3-5),

$$
\left(\omega \mid\left[L(1), a_{(0)}\right] b\right)=4(a \mid b)-2(L(1) a \mid L(1) b)+\left(\omega \mid(L(1) a)_{(0)} b\right) .
$$

On the other hand, by (1) for $(m, n)=(3,0)$ and the Virasoro relation,

$$
\begin{aligned}
\left(\omega \mid\left[L(1), a_{(0)}\right] b\right) & =\left(L(-1) \omega \mid a_{(0)} b\right)-\left(\omega \mid a_{(0)} L(1) b\right) \\
& =4(a \mid b)-2(L(1) a \mid L(1) b)-\left(\omega \mid a_{(0)} L(1) b\right) .
\end{aligned}
$$

We see from (3-5), (3-7), and (3-8) that $\left(\omega \mid(L(1) a)_{(0)} b\right)$ is computed as follows:

$$
\begin{aligned}
\left(\omega \mid(L(1) a)_{(0)} b\right) & =-\left(\omega \mid a_{(0)} L(1) b\right) \\
& =-\left(\mathbf{1} \mid\left[L(2), a_{(0)}\right] L(1) b\right) \\
& =-3\left(\left(\mathbf{1} \mid a_{(2)} L(1) b\right)+\left(\mathbf{1} \mid(L(1) a)_{(1)} L(1) b\right)\right) \\
& =-3(L(1) a \mid L(1) b)+3(L(1) a \mid L(1) b)=0 .
\end{aligned}
$$

Therefore (2) holds by (3-6).

Let $V$ be an SVOA with minimal conformal weight $\mu \in\left\{\frac{1}{2}, 1, \frac{3}{2}, 2\right\}$. We give trace formulae of the zero-modes of elements of $V_{2}$ on $V_{\mu}$ by using the same method as in [Matsuo 2001, Section 2.3]. Let $a, b \in V_{2}$, and $w \in V_{\mu}$. In general, by the Borcherds-Jacobi identity (see [Kac 1998]), for $u, v \in V^{0}$ and $p, q, r \in \mathbb{Z}$,

$$
\begin{aligned}
\sum_{\ell \geq 0}\left(\begin{array}{l}
p \\
\ell
\end{array}\right)\left(u_{(r+\ell)} v\right)_{(p+q-\ell)} & \\
= & \sum_{\ell \geq 0}(-1)^{\ell}\left(\begin{array}{l}
r \\
\ell
\end{array}\right)\left(u_{(p+r-\ell)} v_{(q+\ell)}-(-1)^{r} v_{(q+r-\ell)} u_{(p+\ell)}\right) .
\end{aligned}
$$


By (3-9) for $p=2, q=1$, and $r=-1$,

$$
a_{(1)} b_{(1)} w=\sum_{\ell=0}^{2}\left(\begin{array}{l}
2 \\
\ell
\end{array}\right)\left(a_{(-1+\ell)} b\right)_{(3-\ell)} w-a_{(-1)} b_{(3)} w-b_{(-1)} a_{(3)} w .
$$

Therefore,

$$
\left.\operatorname{tr}\right|_{V_{\mu}} o(a) o(b)=\left.\sum_{\ell=0}^{2}\left(\begin{array}{l}
2 \\
\ell
\end{array}\right) \operatorname{tr}\right|_{V_{\mu}} o\left(\pi\left(a_{(-1+\ell)} b\right)\right)-2 \delta_{\mu, 2}(a \mid b)
$$

if $V_{\mu}$ forms a conformal 4-design based on the even part. Then, by Lemma 3.7 one can compute the trace because $\pi\left(a_{(-\ell+1)} b\right) \in\left(V_{\omega}\right)_{4-\ell}$, yielding

$$
\begin{aligned}
\left.\operatorname{tr}\right|_{V_{\mu}} o(L(-4) \mathbf{1}) & =3 \mu d_{\mu}, & \left.\operatorname{tr}\right|_{V_{\mu}} o\left(L(-2)^{2} \mathbf{1}\right) & =\mu d_{\mu}(\mu+2)+c \delta_{\mu, 2}, \\
\left.\operatorname{tr}\right|_{V_{\mu}} o(L(-3) \mathbf{1}) & =-2 \mu d_{\mu}, & \left.\operatorname{tr}\right|_{V_{\mu}} o(L(-2) \mathbf{1}) & =\mu d_{\mu} .
\end{aligned}
$$

Note that the cases $\mu \in \frac{1}{2}+\mathbb{Z}_{\geq 1} \cup\{2\}$ have already been obtained in [Matsuo 2001; Yamauchi 2014].

Proposition 3.8 [Matsuo 2001, Theorem 2.1; Yamauchi 2014, Theorem 1]. Let V be an SVOA of central charge $c$ with minimal conformal weight $\mu \in\left\{\frac{1}{2}, 1, \frac{3}{2}, 2\right\}$.

(1) If $V_{\mu}$ forms a conformal 2-design, then for $a \in V_{2}$,

$$
\left.\operatorname{tr}\right|_{V_{\mu}} o(a)=\frac{2 \mu d_{\mu}}{c}(a \mid \omega) \text {. }
$$

(2) If $V_{\mu}$ forms a conformal 4-design, then for $a, b \in V_{2}$,

$$
\begin{array}{r}
\left.\operatorname{tr}\right|_{V_{\mu}} o(a) o(b)=\frac{2\left(\mu d_{\mu}(22 \mu-c)-5 c^{2} \delta_{\mu, 2}\right)}{c(5 c+22)}(a \mid b) \\
-\frac{2\left(\mu d_{\mu}(c+6+8 \mu)+8 c \delta_{\mu, 2}\right)}{c(5 c+22)}(L(1) a \mid L(1) b) \\
\quad+\frac{4\left(\mu d_{\mu}(5 \mu+1)+5 c \delta_{\mu, 2}\right)}{c(5 c+22)}(a \mid \omega)(b \mid \omega) .
\end{array}
$$

Remark 3.9. The reason why we consider the cases where $\mu \in\left\{\frac{1}{2}, 1, \frac{3}{2}, 2\right\}$ is that the trace of $o(a)$ on $V_{\mu}$ for $a \in V_{2}$ is a multiple of $\mu d_{\mu}$ because $V_{2}=\left(V_{\omega}\right)_{2}$ if $\mu>2$. Hence, we consider trace formulae on $V_{\mu}$ in the cases $\mu \leq 2$ only.

Set $d_{\mu}^{e}(k):=\operatorname{dim} W_{\mu}^{e}(k)$, where $W_{\mu}^{e}(k)$ is defined in (2-1). The following corollary holds. It has already been mentioned in the introduction of [loc. cit.] for $\mu=2$.

Corollary 3.10. Let $V$ be an SVOA with minimal conformal weight $\mu \in\left\{\frac{1}{2}, 1, \frac{3}{2}\right\}$, and e an Ising vector. If $V_{\mu}$ forms a conformal 4-design based on $V^{0}$, then

$$
d_{\mu}^{e}(0)=\frac{d_{\mu}(c(5 c+22-61 \mu)+2 \mu(196 \mu-95))}{c(5 c+22)} .
$$


Also,

$$
d_{\mu}^{e}\left(\frac{1}{2}\right)=\frac{\mu d_{\mu}(56 \mu-3 c-2)}{c(5 c+22)} \quad \text { and } \quad d_{\mu}^{e}\left(\frac{1}{16}\right)=\frac{64 \mu d_{\mu}(c+3-7 \mu)}{c(5 c+22)} .
$$

Proof. Obviously, $\left.\operatorname{tr}\right|_{V_{\mu}} o(\mathbf{1})=d_{\mu}$. Note that $L(1) e=0$ since $V$ is of CFT-type; see [Yamauchi 2004, Lemma 8.1.2]. Since $(\omega \mid e)=(e \mid e)=\frac{1}{4}$, by Proposition 3.8,

$$
\left.\operatorname{tr}\right|_{V_{\mu}} o(e)=\frac{\mu d_{\mu}}{2 c} \text { and }\left.\operatorname{tr}\right|_{V_{\mu}} o(e)^{2}=\frac{\mu d_{\mu}(49 \mu-2 c+1)}{4 c(5 c+22)} .
$$

By Lemma 2.1,

$$
\left[\begin{array}{ccc}
1 & 1 & 1 \\
0 & \frac{1}{2} & \frac{1}{16} \\
0 & \left(\frac{1}{2}\right)^{2} & \left(\frac{1}{16}\right)^{2}
\end{array}\right]\left[\begin{array}{c}
d_{\mu}^{e}(0) \\
d_{\mu}^{e}\left(\frac{1}{2}\right) \\
d_{\mu}^{e}\left(\frac{1}{16}\right)
\end{array}\right]=\left[\begin{array}{c}
d_{\mu} \\
\frac{\mu d_{\mu}}{2 c} \\
\frac{\mu d_{\mu}(49 \mu-2 c+1)}{4 c(5 c+22)}
\end{array}\right] .
$$

Therefore, we obtain this corollary by direct computation.

The following corollary is obtained from (3-10) immediately.

Corollary 3.11. Let $V$ be an SVOA of central charge $c$ with minimal conformal weight $\mu \in\left\{\frac{1}{2}, 1, \frac{3}{2}\right\}$, and e an Ising vector of $V$. Assume that $V_{\mu}$ forms a conformal 4-design based on the even part. Then $c=7 \mu-3$ if and only if $d_{\mu}^{e}\left(\frac{1}{16}\right)=0$.

An $\mathbb{R}$-form $W$ of an SVOA $V$ is an $\mathbb{R}$-subalgebra of $V$ with the same Virasoro element such that $V \cong \mathbb{C} \otimes W$. As an application of the trace formulae, we have the following theorem.

Theorem 3.12. Let $V$ be an SVOA with minimal conformal weight $\frac{1}{2}$ and let $W$ be an $\mathbb{R}$-form which has a positive definite invariant bilinear form. If $V_{1 / 2}$ forms a conformal 4-design based on the even part, then $V$ is isomorphic to

$$
L\left(\frac{1}{2}, 0\right) \oplus L\left(\frac{1}{2}, \frac{1}{2}\right) .
$$

Remark 3.13. [Höhn 2008, Theorem 4.1(a)] shows that $V$, as in Theorem 3.12, is isomorphic to $L\left(\frac{1}{2}, 0\right) \oplus L\left(\frac{1}{2}, \frac{1}{2}\right)$ if the minimal conformal weight space forms a conformal 6-design. Hence, Theorem 3.12 is more general than the theorem in that reference.

Proof of Theorem 3.12. Since the bilinear form is positive definite on $W$, we can take an orthogonal basis $\left\{x^{i}\right\}_{i=1}^{d_{1 / 2}}$ of $W_{1 / 2}$ such that $\left(x^{i} \mid x^{j}\right)=\frac{1}{2} \delta_{i j}$. Since $V \cong \mathbb{C} \otimes W$, $\left\{x^{i}\right\}_{i=1}^{d_{1 / 2}}$ is also a basis of $V_{1 / 2}$. Set

$$
e^{i}:=-x_{(-2)}^{i} x^{i} \quad \text { and } \quad L^{e^{i}}(n)=e_{(n+1)}^{i}
$$

for each $1 \leq i \leq d_{1 / 2}$ and $n \in \mathbb{Z}$. Then for each $1 \leq i \leq d_{1 / 2}$ we can check by direct computation that $\left\{L^{e^{i}}(n)\right\}_{n \in \mathbb{Z}}$ satisfies the Virasoro relation with central charge $\frac{1}{2}$. 
Since $e^{1}, \ldots, e^{d_{1 / 2}}$ belong to $W$, the vectors are Ising vectors; see [Miyamoto 1996b, Section 6, p. 540]. Also by direct computation,

$$
L^{e^{i}}(0) x^{j}=\frac{1}{2} \delta_{i j} x^{j}
$$

for $1 \leq i, j \leq d_{1 / 2}$. Because $\left\{x^{i}\right\}_{i=1}^{d_{1 / 2}}$ is a basis of $V_{1 / 2}$, the central charge of $V$ is $\frac{1}{2}$ by Corollary 3.11. Then by Proposition 3.8 we also have $d_{1 / 2}=1$ since $\left.\operatorname{tr}\right|_{V_{1 / 2}} o\left(e^{i}\right)=\frac{1}{2}$. Since the central charge is $\frac{1}{2}$ and $\omega$ is the Virasoro element of $W$, we may conclude that $\omega$ is an Ising vector of $V$. Because the $L(0)$-weights of $V$ are half-integers, $V=V[0] \oplus V\left[\frac{1}{2}\right]$, where $V[k]$ is the $V_{\omega}$-submodule of $V$ defined at the beginning of Section 3. Therefore,

$$
V=V_{\omega} \oplus V\left[\frac{1}{2}\right] \cong L\left(\frac{1}{2}, 0\right) \oplus L\left(\frac{1}{2}, \frac{1}{2}\right)
$$

because $V[0]=V_{\omega}$ and $d_{1 / 2}=1$.

\section{Conformal designs and code SVOAs}

In this section, we first review the notion of binary codes and combinatorial designs. Next, we recall the definition of code SVOAs, and classify the code SVOAs whose minimal conformal weight spaces form conformal 4-designs.

Binary codes. A binary code $C$ of length $n$ is a subspace of $\mathbb{F}_{2}^{n}$. The support $\operatorname{supp}(x)$ and the weight $\operatorname{wt}(x)$ of $x=\left(x_{1}, \ldots, x_{n}\right) \in \mathbb{F}_{2}^{n}$ are defined by

$$
\operatorname{supp}(x):=\left\{1 \leq i \leq n \mid x_{i} \neq 0\right\} \quad \text { and } \quad \operatorname{wt}(x):=\# \operatorname{supp}(x),
$$

respectively. A binary code $C$ is said to be even if wt $(c) \in 2 \mathbb{Z}$ for all $c \in C$. Let $\left(0^{n}\right)$ and $\left(1^{n}\right)$ denote the vectors $(0, \ldots, 0) \in \mathbb{F}_{2}^{n}$ and $(1, \ldots, 1) \in \mathbb{F}_{2}^{n}$, respectively. The minimum weight of $C$ is $\min \left\{\operatorname{wt}(c) \mid c \in C \backslash\left\{\left(0^{n}\right)\right\}\right\}$ if $C \neq\left\{\left(0^{n}\right)\right\}$ and $\infty$ if $C=\left\{\left(0^{n}\right)\right\}$. For $x=\left(x_{1}, \ldots, x_{n}\right), y=\left(y_{1}, \ldots, y_{n}\right) \in \mathbb{F}_{2}^{n}$, let $x * y$ denote the vector $\left(x_{1} y_{1}, \ldots, x_{n} y_{n}\right) \in \mathbb{F}_{2}^{n}$. For $C$ a binary code with minimum weight $d \neq \infty$,

$$
\# C \leq 2^{n} / \sum_{i=0}^{\left\lfloor\frac{d-1}{2}\right\rfloor}\left(\begin{array}{l}
n \\
i
\end{array}\right),
$$

where $\left\lfloor\frac{1}{2}(d-1)\right\rfloor$ is the largest integer not greater than $\frac{1}{2}(d-1)$. The upper bound of $\# C$ is called the sphere-packing bound of $C$; see [Assmus and Key 1992, Theorem 2.1.3]. It is easy to see that $d$ is an odd integer if equality holds in (4-1).

Set $\widehat{\mathcal{H}}_{2}:=\left\{\left(0^{4}\right),\left(1^{4}\right)\right\}$. Define the binary code $\hat{\mathcal{H}}_{m}$ for $m \in \mathbb{Z}_{\geq 3}$ by

$$
\left\{(u, u+v) \mid u \in \mathcal{E}_{2^{m-1}}, v \in \widehat{\mathcal{H}}_{m-1}\right\}
$$


where $\mathcal{E}_{\ell}$ is the set of all even weight vectors in $\mathbb{F}_{2}^{\ell}$. Set

$$
\mathcal{H}_{m}:=\left\{\left(c_{1}, \ldots, c_{2^{m}-1}\right) \mid\left(c_{1} \ldots, c_{2^{m}}\right) \in \widehat{\mathcal{H}}_{m}\right\} \subset \mathbb{F}_{2}^{2^{m}-1}
$$

for $m \in \mathbb{Z}_{\geq 2}$. The binary codes $\mathcal{H}_{m}$ and $\hat{\mathcal{H}}_{m}$ are called the Hamming code of length $2^{m}-1$ and the extended Hamming code of length $2^{m}$, respectively; see [MacWilliams and Sloane 1977, Chapters 1 and 13]. Denote by $E\left(\mathcal{H}_{m}\right)$ the even subcode of $\mathcal{H}_{m}$. Note that the dimensions of $\mathcal{H}_{m}$ and $\hat{\mathcal{H}}_{m}$ are $2^{m}-m-1$. The following lemmas are obtained by a basic method of algebraic coding theory. For the reader's convenience we include the proof.

Lemma 4.1. Let $m \in \mathbb{Z}_{\geq 2}$ and $D$ a binary code of length $2^{m}$ whose minimum weight is greater than or equal to 3 . If $D$ has a subcode equivalent to $\widehat{\mathcal{H}}_{m}$, then $D$ is equivalent to $\hat{\mathcal{H}}_{m}$.

Proof. By (4-1),

$$
\# D \leq 2^{2^{m}} / \sum_{i=0}^{1}\left(\begin{array}{c}
2^{m} \\
i
\end{array}\right)<2^{2^{m}-m} .
$$

Hence $\operatorname{dim} D$ is less than or equal to $2^{m}-m-1$. Since $\operatorname{dim} \hat{\mathcal{H}}_{m}=2^{m}-m-1$, the assertion holds.

Lemma 4.2. Let $m \in \mathbb{Z}_{\geq 2}$ and $D$ a binary code of length $2^{m}-1$ whose minimum weight is greater than or equal to 3 . If $D$ has a subcode equivalent to $E\left(\mathcal{H}_{m}\right)$, then $D$ is equivalent to $E\left(\mathcal{H}_{m}\right)$ or $\mathcal{H}_{m}$.

Proof. We see from (4-1) that $\# D \leq 2^{2^{m}-1} / \sum_{i=0}^{1}\left(\left(^{2^{m}-1} i\right)=2^{2^{m}-1-m}\right.$. Hence $\operatorname{dim} D$ is $2^{m}-1-m$ or $2^{m}-2-m$ because $D$ has a subcode equivalent to $E\left(\mathcal{H}_{m}\right)$. Clearly, $D$ is equivalent to $E\left(\mathcal{H}_{m}\right)$ if $\operatorname{dim} D$ is $2^{m}-2-m$. If $\operatorname{dim} D$ equals $2^{m}-1-m$, then equality holds in (4-1). As already mentioned before, the minimum weight of $D$ must be an odd integer, and hence it must be 3 . Thus $D$ is a binary code of length $2^{m}-1$ whose dimension and minimum weight are $2^{m}-1-m$ and 3 , respectively. It is known that such a code is equivalent to $\mathcal{H}_{m}$; see [MacWilliams and Sloane 1977, Chapter 1, Section 7, Problem (28)]. Therefore, this lemma holds.

Combinatorial designs and binary codes. Set $\Omega_{n}:=\{1, \ldots, n\}$. Let $k$ be a nonnegative integer such that $k \leq n$. Denote the set of all $k$-subsets of $\Omega_{n}$ by $\left(\begin{array}{c}\Omega_{n} \\ k\end{array}\right)$. Let $\mathcal{B}$ be a subset of $\left(\begin{array}{c}\Omega_{n} \\ k\end{array}\right)$. A pair $\left(\Omega_{n}, \mathcal{B}\right)$ is a $t-(n, k, \lambda)$-design if there exists a constant $\lambda$ such that $\#\{B \in \mathcal{B} \mid X \subset B\}=\lambda$ for all $X \in\left(\begin{array}{c}\Omega_{n} \\ t\end{array}\right)$. For $D \subset \mathbb{F}_{2}^{n}$, set $D(k):=\{u \in D \mid \operatorname{wt}(u)=k\}$. We often say that $\left(\Omega_{n}, D(k)\right)$ is a $t$ - $(n, k, \lambda)$-design

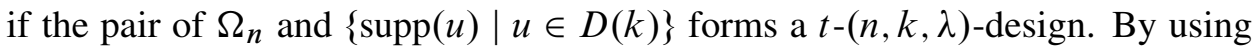
a basic method for algebraic coding theory, the following proposition holds. We include the proof for the reader's convenience. 
Proposition 4.3. Let $m \in \mathbb{Z}_{\geq 2}$ and $C$ a binary code of length $2^{m}$ with minimum weight 4. If $\left(\Omega_{2^{m}}, C(4)\right)$ forms a 3- $\left(2^{m}, 4,1\right)$-design, then $C$ is equivalent to $\hat{\mathcal{H}}_{m}$. Analogously, let $C$ be a binary code of length $2^{m}-1$ with minimum weight 3 . If $\left(\Omega_{2^{m}-1}, C(3)\right)$ forms a 2-( $\left.2^{m}-1,3,1\right)$-design, then $C$ is equivalent to $\mathcal{H}_{m}$.

Proof. We show the $\hat{\mathcal{H}}_{m}$ case only because the $\mathcal{H}_{m}$ case is obtained by the same method. If we show that $\#\left(\mathbb{F}_{2}^{2 m} / C\right)=2^{m+1}$, then we obtain the statement because a binary code of length $2^{m}$ whose dimension and minimum weight are $2^{m}-1-m$ and 4, respectively, is equivalent to $\hat{\mathcal{H}}_{m}$ [MacWilliams and Sloane 1977, Chapter 1, Section 9, Problem (41)]. Let $u \in \mathbb{F}_{2}^{2^{m}}$ such that $\operatorname{wt}(u)>2$. Then there exists $v \in C(4)$ such that $\operatorname{wt}(u+v) \leq \operatorname{wt}(u)-2$ because $\left(\Omega_{2^{m}}, C(4)\right)$ is a 3- $\left(2^{m}, 4,1\right)$ design. Hence, every element of $\mathbb{F}_{2}^{2^{m}} / C$ is represented by an element of weight at most 2. Also, since the weight of the sum of vectors $x, x^{\prime} \in \mathbb{F}_{2}^{2^{m}}$ such that $\operatorname{wt}(x) \leq 1, \operatorname{wt}\left(x^{\prime}\right) \leq 2$, and $x \neq x^{\prime}$ is less than 4 and the minimum weight of $C$ is 4 , $x+C$ and $x^{\prime}+C$ are distinct. Set $X_{i}:=\left\{y+C \in \mathbb{F}_{2}^{2^{m}} / C \mid \operatorname{wt}(y)=i\right\}$. By the argument above, we have

$$
\mathbb{F}_{2}^{2 m} / C=X_{0} \amalg X_{1} \amalg X_{2}, \quad \# X_{0}=1, \quad \text { and } \quad \# X_{1}=2^{m} .
$$

Hence, it is sufficient to show that $\# X_{2}=2^{m}-1$. Let $y+C \in X_{2}$. It is easy to check that

$$
(y+C)(2)=\{y\} \amalg\{y+c \mid c \in C(4) \text { such that } \operatorname{supp}(y) \subset \operatorname{supp}(c)\} .
$$

Hence, $\#(y+C)(2)=2^{m-1}$ because $\left(\Omega_{2^{m}}, C(4)\right)$ is also a $2-\left(2^{m}, 4,2^{m-1}-1\right)$ design [op. cit., Chapter 2, Section 5, Theorem 9]. Since

$$
\mathbb{F}_{2}^{2^{m}}(2)=\coprod_{z+C \in X_{2}}(z+C)(2),
$$

we have $\# X_{2}=\frac{1}{2^{m-1}}\left(\begin{array}{c}2^{m} \\ 2\end{array}\right)=2^{m}-1$, completing the proof of this proposition.

In order to prove our main result, we need the following two lemmas.

Lemma 4.4. Let $t \in \mathbb{Z}_{\geq 2}$ and let $C$ be a binary code of length $n \geq 3$ with minimum weight $t+1$. Then the cardinality of $C(t+1)$ is at most $\frac{1}{t+1}\left(\begin{array}{c}n \\ t\end{array}\right)$. Moreover, equality holds in the inequality if and only if $\left(\Omega_{n}, C(t+1)\right)$ forms a $t-(n, t+1,1)$-design.

Proof. Consider the cardinality of

$$
S:=\left\{X \in\left(\begin{array}{c}
\Omega_{n} \\
t
\end{array}\right) \mid \text { there exists } u \in C(t+1) \text { such that } X \subset \operatorname{supp}(u)\right\} .
$$

Since $t \geq 2$ and the minimum weight of $C$ is $t+1$, for $X \in\left(\begin{array}{c}\Omega_{n} \\ t\end{array}\right)$ the cardinality of $\{u \in C(t+1) \mid X \subset \operatorname{supp}(u)\}$ is at most 1. Hence

$$
S=\coprod_{u \in C(t+1)}\left(\begin{array}{c}
\operatorname{supp}(u) \\
t
\end{array}\right) .
$$


By (4-2),

$$
\left(\begin{array}{l}
n \\
t
\end{array}\right) \geq \# S=\sum_{u \in C(t+1)} \#\left(\begin{array}{c}
\operatorname{supp}(u) \\
t
\end{array}\right)=(t+1) \# C(t+1) .
$$

Therefore, the first claim of this lemma holds. Also, from (4-2), that equality holds in the inequality if and only if $\left(\begin{array}{c}\Omega_{n} \\ t\end{array}\right)=S$. Hence we have the second claim because $\left(\begin{array}{c}\Omega_{n} \\ t\end{array}\right)=S$ implies that $\left(\Omega_{n}, C(t+1)\right)$ forms a $t$ - $(n, t+1,1)$-design.

Lemma 4.5. Let $C$ be an even code of length $2^{m}-1(m \geq 2)$ with minimum weight 4 . If $\left(\Omega_{2^{m}-1}, C(4)\right)$ forms a $2-\left(2^{m}-1,4,2^{m-1}-2\right)$-design, then $C \cong E\left(\mathcal{H}_{m}\right)$.

Proof. Set $D:=\left\langle C,\left(1^{2^{m}-1}\right)\right\rangle_{\mathbb{F}_{2}}=C \amalg\left(\left(1^{2^{m}-1}\right)+C\right)$. Note that if we show that the minimum weight of $D$ is 3 and $\left(\Omega_{2^{m}-1}, D(3)\right)$ forms a $2-\left(2^{m}-1,3,1\right)$-design, then this lemma follows from Lemma 4.2 since the even subcode of $D$ is $C$.

First we show that the minimum weight of $D$ is 3 . Fix $X \in\left(\Omega_{2}^{2 m}-1\right)$. Set $C_{X}:=\{u \in C(4) \mid X \subset \operatorname{supp}(u)\}$ and $w_{X}:=\sum_{u \in C_{X}} u$. Let $u, v \in C_{X}$ such that $u \neq v$. Then $\operatorname{supp}(u) \cap \operatorname{supp}(v)=X$ because the minimum weight of $C$ is 4 . Since $\left(\Omega_{2^{m}-1}, C(4)\right)$ forms a $2-\left(2^{m}-1,4,2^{m-1}-2\right)$-design, the cardinality of $C_{X}$ is $2^{m-1}-2$, and hence we have $\operatorname{wt}\left(w_{X}\right)=2^{m}-4$ and $\left(1^{2^{m}-1}\right)+w_{X} \in D(3)$. If we suppose that $D$ has a weight-1 vector $v$, then $\left(1^{2^{m}-1}\right)+w_{Y}+v \in D(2)$ for $Y \in\left(\begin{array}{c}\Omega_{2} m-1 \\ 2\end{array}\right)$ such that $\operatorname{supp}(v) \subset Y$, which contradicts $D(2)=C(2)=\varnothing$. Thus, the minimum weight of $D$ is 3 .

Next we show that $\left(\Omega_{2^{m}-1}, D(3)\right)$ forms a $2-\left(2^{m}-1,3,1\right)$-design. Set $z_{X}:=$ $\left(1^{2^{m}-1}\right)+w_{X}$ for $X \in\left(\begin{array}{c}\Omega_{2} m_{-1} \\ 2\end{array}\right)$. Then wt $\left(w_{X}+w_{Y}\right)=6-2 \mathrm{wt}\left(z_{X} * z_{Y}\right)$ for $X, Y \in\left(\begin{array}{c}\Omega_{2} m-1 \\ 2\end{array}\right)$. Since $C(2)=\varnothing$, we have $w t\left(z_{X} * z_{Y}\right) \leq 1$ if $w_{X} \neq w_{Y}$, and $\operatorname{wt}\left(z_{X} * z_{Y}\right)=3$ if $w_{X}=w_{Y}$. Because the support of $z_{X} * z_{Y}$ is

$$
\left(\Omega_{2^{m}-1} \backslash \operatorname{supp}\left(w_{X}\right)\right) \cap\left(\Omega_{2^{m}-1} \backslash \operatorname{supp}\left(w_{Y}\right)\right),
$$

we have $w_{X}=w_{Y}$ if and only if $Y \subset \Omega_{2^{m_{-1}}} \backslash \operatorname{supp}\left(w_{X}\right)$. By this argument, $\#\left\{w_{X} \mid X \in\left(\begin{array}{c}\Omega_{2} m-1 \\ 2\end{array}\right)\right\}$ is exactly $\frac{1}{3}\left(2^{m}-1\right)$. Hence $\# D(3) \geq \frac{1}{3}\left(2^{2}-1\right)$. We see from Lemma 4.4 that the assertion holds.

Code SVOAs. Let $X$ be the free fermionic SVOA of central charge $\frac{1}{2}$, i.e., $X=$ $L\left(\frac{1}{2}, 0\right) \oplus L\left(\frac{1}{2}, \frac{1}{2}\right)$. Set $X^{k}:=L\left(\frac{1}{2}, \frac{k}{2}\right)$ for $k=0,1$. Then $X^{\otimes n}$ is an SVOA as a tensor product of SVOAs. Set $V^{\alpha}:=X^{\alpha_{1}} \otimes \cdots \otimes X^{\alpha_{n}}$ for $\alpha=\left(\alpha_{1}, \ldots, \alpha_{n}\right) \in \mathbb{F}_{2}^{n}$. Note that $V^{\alpha}$ is a $V^{\left(0^{n}\right)}$-module. For a binary code $C$ of length $n$, set

$$
V_{C}:=\bigoplus_{\alpha \in C} V^{\alpha}
$$

which is a sub-SVOA of $X^{\otimes n}$. The SVOA $V_{C}$ is called the code SVOA associated to $C$; see [Miyamoto 1996a; Lam et al. 2007] for details. We remark that the central charge of $V_{C}$ is half of the length of $C$. Let $u^{0}=\mathbf{1}$ be the vacuum vector 
of $X^{0}$ and $u^{1}$ a highest weight vector of $X^{1}$ such that $u_{(-2)}^{1} u^{1}=2 \omega$, where $\omega$ is the Virasoro element of $X^{0}$. For $\alpha=\left(\alpha_{1}, \ldots, \alpha_{n}\right) \in \mathbb{F}_{2}^{n}$, set

$$
u^{\alpha}:=u^{\alpha_{1}} \otimes \cdots \otimes u^{\alpha_{n}} \in V^{\alpha} .
$$

Note that $u^{\alpha}$ is a highest weight vector of $V^{\alpha}$. For $1 \leq i \leq n$, set

$$
e^{i}:=\mathbf{1} \otimes \cdots \otimes \omega \otimes \cdots \otimes \mathbf{1} \in V_{C},
$$

where the Virasoro element $\omega$ of $X^{0}$ is the $i$-th tensor factor. It is known that $e^{i}$ is an Ising vector of $\sigma$-type; see [Miyamoto 1996a]. Let $(\cdot \mid \cdot)$ be the invariant bilinear form on $V_{C}$ such that $(\mathbf{1} \mid \mathbf{1})=1$. Then $\left(e^{i} \mid e^{j}\right)=\frac{1}{4} \delta_{i j}$ obviously holds.

Set $N:=\bigoplus_{1 \leq i, j \leq 2 n} \mathbb{Z}\left(x_{i}+x_{j}\right)$, where $\left\{x_{i}\right\}_{i=1}^{2 n}$ is an orthonormal basis of $\mathbb{R}^{2 n}$. Let $\left(V_{N}\right)_{\mathbb{R}}$ be the lattice VOA over $\mathbb{R}$ associated to $N$. In [Miyamoto 2004], it was proved that if a binary code $C$ of length $n$ is even, then the code VOA over $\mathbb{R}$ is embedded into the VOA $\left(V_{N}^{+}\right)_{\mathbb{R}} \oplus \sqrt{-1}\left(V_{N}^{-}\right)_{\mathbb{R}}$, where $\left(V_{N}^{ \pm}\right)_{\mathbb{R}} \subset\left(V_{N}\right)_{\mathbb{R}}$ is the eigenspace of a lift of the -1 isometry of $N$ with eigenvalue \pm 1 , respectively. The VOA $\left(V_{N}^{+}\right)_{\mathbb{R}} \oplus \sqrt{-1}\left(V_{N}^{-}\right)_{\mathbb{R}}$ has a positive definite invariant bilinear form; see [op. cit., Proposition 2.7]. Replacing $N$ by $L:=\bigoplus_{i=1}^{2 n} \mathbb{Z} x_{i}$, one can show the case that $C$ has an odd weight vector, and hence the following holds.

Proposition 4.6 [Miyamoto 2004, Corollary 3.6]. Let $C$ be a binary code. Then $V_{C}$ has an $\mathbb{R}$-form which has a positive definite invariant bilinear form. In particular, $V_{C}$ satisfies the assumptions in Section 3 on page 126.

Conformal 4-designs and code SVOAs. Let $C$ be a binary code and $\mu$ the minimal conformal weight of the code SVOA $V_{C}$. Assume that $\mu<\infty$. We show that $C$ is equivalent to $\left\{\left(0^{1}\right),\left(1^{1}\right)\right\}, \widehat{\mathcal{H}}_{3}, \mathcal{E}_{8}, E\left(\mathcal{H}_{4}\right), \mathcal{H}_{4}$, or $\hat{\mathcal{H}}_{4}$ if $\left(V_{C}\right)_{\mu}$ forms a conformal 4-design based on $V_{C}^{0}$. The next lemma plays an important role in our main result. Lemma 4.7. Let $C$ be a binary code of length $n$ and $\mu$ the minimal conformal weight of $V_{C}$. Assume that $\mu \in\left\{1, \frac{3}{2}, 2\right\}$. If $\left(V_{C}\right)_{\mu}$ forms a conformal 4-design based on $V_{C}^{0}$, then $\left(\Omega_{n}, C(2 \mu)\right)$ forms a $2-(n, 2 \mu, v)$-design, where

$$
\nu=\frac{4 \mu(5 \mu+1) \# C(2 \mu)+98 n \delta_{\mu, 2}}{n(5 n+44)} .
$$

Proof. A basis of $\left(V_{C}\right)_{\mu}$ is given by

$$
\begin{cases}\left\{u^{\alpha} \mid \alpha \in C(2 \mu)\right\} & \text { if } \mu=1, \frac{3}{2}, \\ \left\{e^{i} \mid 1 \leq i \leq n\right\} \cup\left\{u^{\alpha} \mid \alpha \in C(4)\right\} & \text { if } \mu=2,\end{cases}
$$

where $e^{i}$ and $u^{\alpha}$ are defined in Section 4 on page 137. Let $i, j \in \Omega_{n}$ with $i \neq j$. Due to $\left(e^{i} \mid e^{j}\right)=0, L(1) e^{i}=L(1) e^{j}=0$, and Proposition 3.8(2),

$$
\left.\operatorname{tr}\right|_{\left(V_{C}\right)_{\mu}} o\left(e^{i}\right) o\left(e^{j}\right)=\frac{\mu d_{\mu}(5 \mu+1)+5 c \delta_{\mu, 2}}{4 c(5 c+22)},
$$


where $c$ is the central charge of $V_{C}$, i.e., $c=\frac{n}{2}$ and

$$
d_{\mu}=\operatorname{dim}\left(V_{C}\right)_{\mu}=\# C(2 \mu)+n \delta_{\mu, 2} .
$$

On the other hand,

$$
\left.\operatorname{tr}\right|_{\left(V_{C}\right)_{\mu}} o\left(e^{i}\right) o\left(e^{j}\right)=\frac{1}{4} \#\{\alpha \in C(2 \mu) \mid i, j \in \operatorname{supp}(\alpha)\}
$$

since

$$
o\left(e^{i}\right) o\left(e^{j}\right) u^{\alpha}=\left\{\begin{array}{ll}
\frac{1}{4} u^{\alpha} & \text { if } i, j \in \operatorname{supp}(\alpha), \\
0 & \text { otherwise, }
\end{array} \quad \text { and } \quad o\left(e^{i}\right) o\left(e^{j}\right) e^{k}=0\right.
$$

for $\alpha \in C$ and $k \in \Omega_{n}$. It follows from (4-4) and (4-5) that

$$
\begin{aligned}
\#\{\alpha \in C(2 \mu) \mid i, j \in \operatorname{supp}(\alpha)\} & =\frac{\mu d_{\mu}(5 \mu+1)+5 c \delta_{\mu, 2}}{c(5 c+22)} \\
& =\frac{4 \mu(5 \mu+1) \# C(2 \mu)+98 n \delta_{\mu, 2}}{n(5 n+44)},
\end{aligned}
$$

concluding the proof.

One of our main results is the following.

Theorem 4.8. Let $C$ be a binary code and $\mu$ the minimal conformal weight of $V_{C}$. Assume that $\mu<\infty$. If $\left(V_{C}\right)_{\mu}$ forms a conformal 4-design based on $V_{C}^{0}$, then $C$ is equivalent to $\left\{\left(0^{1}\right),\left(1^{1}\right)\right\}, \widehat{\mathcal{H}}_{3}, \mathcal{E}_{8}, E\left(\mathcal{H}_{4}\right), \mathcal{H}_{4}$, or $\hat{\mathcal{H}}_{4}$.

Proof. Let $n$ be the length of $C$. Note that $\mu$ must be $\infty$ if $\mu>2$, because $\left(V_{C}\right)_{2}=\left(V_{\omega}\right)_{2}$ implies that $V_{C}=L\left(\frac{1}{2}, 0\right)$ by the construction of code SVOAs. Hence our assumption implies $\mu \leq 2$.

Recall that the Ising vectors $e^{i}$ are of $\sigma$-type. We see from Corollary 3.11 that the central charge is uniquely determined by $\mu$ if $\mu \in\left\{\frac{1}{2}, 1, \frac{3}{2}\right\}$. In case $\mu=\frac{1}{2}$, the central charge is $\frac{1}{2}$, i.e., $n=1$. Moreover the minimum weight of $C$ is 1 because $\left(V_{C}\right) \frac{1}{2}=\operatorname{Span}_{\mathbb{C}}\left\{u^{\alpha} \mid \alpha \in C(1)\right\}$. Hence $C$ must be $\left\{\left(0^{1}\right),\left(1^{1}\right)\right\}$. It follows from Lemma 4.7 that $\left(\Omega_{n}, C(2 \mu)\right)$ forms a $2-(n, 2 \mu, \nu)$-design, where

$$
v=\frac{4 \mu(5 \mu+1) \# C(2 \mu)+98 n \delta_{\mu, 2}}{n(5 n+44)},
$$

if $\mu \in\left\{1, \frac{3}{2}, 2\right\}$. Also, the length of $C$ is 8 if $\mu=1$, and 15 if $\mu=\frac{3}{2}$ by Corollary 3.11. For $\mu=1, C(2)$ is equal to $\mathbb{F}_{2}^{8}(2)$ because $\left(\Omega_{8}, C(2)\right)$ forms a $2-(8,2, \# C(2) / 28)$ design. Hence $\langle C(2)\rangle_{\mathbb{F}_{2}}$ is equivalent to $\mathcal{E}_{8}$, and so is $C$ because the minimum weight of $C$ is 2 . If $\mu=\frac{3}{2}$, then $\left(\Omega_{15}, C(3)\right)$ forms a $2-(15,3, \# C(3) / 35)$-design. More precisely, $\left(\Omega_{15}, C(3)\right)$ forms a $2-(15,3,1)$-design because the minimum weight of $C$ is 3 . Then it follows from Proposition 4.3 that $\langle C(3)\rangle_{\mathbb{F}_{2}}$ is equivalent to $\mathcal{H}_{4}$, and hence we have $C \cong \mathcal{H}_{4}$ by Lemma 4.2. In case $\mu=2$, a list of possible 
pairs of the central charge and $\operatorname{dim}\left(V_{C}\right)_{2}$ has been obtained in [Matsuo 2001, Section 3.2, Table 3.2] since the central charge is a half-integer. Using this list, we obtain another list of possible pairs $n$ and $\# C$ (4) since $\operatorname{dim}\left(V_{C}\right)_{2}=n+\# C(4)$. The two lists are given as follows:

\begin{tabular}{cc|cc}
\hline$c$ & $\operatorname{dim}\left(V_{C}\right)_{2}$ & $c$ & $\operatorname{dim}\left(V_{C}\right)_{2}$ \\
\hline 4 & 22 & $\frac{19}{2}$ & 418 \\
$\frac{15}{2}$ & 120 & 10 & 685 \\
8 & 156 & $\frac{21}{2}$ & 1491 \\
\hline
\end{tabular}

$\Longrightarrow$\begin{tabular}{cc|cc}
\hline$n$ & $\# C(4)$ & $n$ & $\# C(4)$ \\
\hline 8 & 14 & 19 & 399 \\
15 & 105 & 20 & 665 \\
16 & 140 & 21 & 1470 \\
\hline
\end{tabular}

However, $(n, \# C(4))$ cannot be $(19,399),(20,665),(21,1470)$ because these pairs do not satisfy the inequality in Lemma 4.4 . By using Lemma 4.4 again, $\left(\Omega_{8}, C(4)\right)$ (resp., $\left.\left(\Omega_{16}, C(4)\right)\right)$ forms a 3-(8, 4, 1)- design (resp., 3-(16, 4, 1)-design). Hence it follows from Proposition 4.3 that $\langle C(4)\rangle_{\mathbb{F}_{2}}$ is equivalent to $\widehat{\mathcal{H}}_{3}$ (resp., $\widehat{\mathcal{H}}_{4}$ ). Since the minimum weight of $C$ is $4, C$ must be $\widehat{\mathcal{H}}_{3}$ (resp., $\widehat{\mathcal{H}}_{4}$ ) by Lemma 4.1. Also, by (4-6) the pair $\left(\Omega_{15}, C(4)\right)$ forms a $2-(15,4,6)$-design if $(n, \# C(4))=(15,105)$. We see from Lemma 4.5 that $\langle C(4)\rangle_{\mathbb{F}_{2}}$ is equivalent to $E\left(\mathcal{H}_{4}\right)$. Hence $C \cong E\left(\mathcal{H}_{4}\right)$ by Lemma 4.2. This finishes the proof of the theorem.

Remark 4.9. The $\mu=\frac{1}{2}$ case in Theorem 4.8 has been obtained in Theorem 3.12. Nevertheless, we provided a second proof, because this method is easier than the method of Theorem 3.12 when we consider only code SVOAs.

Remark 4.10. It is known that $V_{\mathcal{E}_{8}}$ is isomorphic to the lattice VOA $V_{D_{4}}$ associated to the root lattice of $D_{4}$ type; see [Dong et al. 1998]. It was proved in [Tuite 2009, Theorem 2.8] that a VOA with minimal conformal weight 1 whose 4th Casimir element belongs to $V_{\omega}$ is isomorphic to one of the level 1 affine VOAs associated to the Deligne exceptional series of simple Lie algebras. Thanks to Theorem 3.5, we see that this classification, which contains $V_{D_{4}}$, can be obtained under the condition that $V_{1}$ forms a conformal 4-design. In fact, $V_{D_{4}}$ is the only VOA in the classification by [op. cit.] which is a code SVOA.

\section{Code SVOAs of class $\mathcal{S}^{5}$}

In this section, we show that the code SVOAs associated to the codes in Theorem 4.8 are of class $\mathcal{S}^{5}$. In particular, their minimal conformal weight spaces form conformal 5-designs.

SVOAs of class $\mathcal{S}^{n}$. The notion of SVOAs of class $\mathcal{S}^{n}$ is an analogue of the notion of VOAs of class $\mathcal{S}^{n}$ introduced by Matsuo. 
Definition 5.1 [Matsuo 2001, Definition 1.1]. An SVOA $V=V^{0} \oplus V^{1}$ is said to be of class $\mathcal{S}^{n}$ if $\left(V^{0}\right)^{\operatorname{Aut}(V)}$ coincides with $V_{\omega}$ up to degree $n$ subspace, i.e.,

$$
\left(V^{0}\right)_{m}^{\operatorname{Aut}(V)}=\left(V_{\omega}\right)_{m} \text { for } 0 \leq m \leq n .
$$

Clearly, the definition above is the ordinary definition in [loc. cit.] when $V$ is a VOA. Note that the fixed point subspace of $V^{1}$ is always 0 since an SVOA has an involution which is the identity on the even part and acts as -1 on the odd part.

Proposition 5.2 [Hashikawa and Shimakura 2016, Proposition 2.12]. Let $U$ be a $V O A$ and $W$ a sub-VOA of $U$ with the same Virasoro element $\omega$. Assume that $U$ is completely reducible as a $V_{\omega}$-module. If $W_{n}=\left(V_{\omega}\right)_{n}$, then $W_{n-1}=\left(V_{\omega}\right)_{n-1}$. In particular, an SVOA $V$ is of class $\mathcal{S}^{n}$ if $V^{0}$ is completely reducible as a $V_{\omega}$-module and $\left(V^{0}\right)_{n}^{\operatorname{Aut}(V)}=\left(V_{\omega}\right)_{n}$.

The following lemma holds.

Lemma 5.3. Let $V=V^{0} \oplus V^{1}$ be an SVOA of class $\mathcal{S}^{n}$. Then:

(1) The even part $V^{0}$ is also of class $\mathcal{S}^{n}$.

(2) The minimal conformal weight space of $V$ forms a conformal $n$-design based on $V^{0}$.

Proof. Since Aut $(V)$ preserves $V^{0}$, there exists a group homomorphism

$$
\varphi: \operatorname{Aut}(V) \rightarrow \operatorname{Aut}\left(V^{0}\right),\left.\quad g \mapsto g\right|_{V^{0}} .
$$

Then we have $\operatorname{Aut}(V) / \operatorname{ker} \varphi \cong \operatorname{Im} \varphi \subset \operatorname{Aut}\left(V^{0}\right)$. Hence (1) is proved because $\left(V^{0}\right)_{n}^{\operatorname{Aut}(V)}$ contains $\left(V^{0}\right)_{n}^{\operatorname{Aut}\left(V^{0}\right)}$. Also, since $\kappa_{\mu}^{n} \in\left(V^{0}\right)_{n}^{\operatorname{Aut}(V)}=\left(V_{\omega}\right)_{n}$, we obtain (2) by Theorem 3.5.

Automorphism groups of code SVOAs. The symmetric group $S_{n}$ of degree $n$ acts on $\mathbb{F}_{2}^{n}$ by $\sigma\left(x_{1}, \ldots, x_{n}\right):=\left(x_{\sigma^{-1}(1)}, \ldots, x_{\sigma^{-1}(n)}\right)$ for $\sigma \in S_{n}$ and $\left(x_{1}, \ldots, x_{n}\right) \in \mathbb{F}_{2}^{n}$. Let $C$ be a binary code of length $n$. An element $\sigma \in S_{n}$ is called an automorphism of $C$ if $\sigma(C)=C$. Let $\operatorname{Aut}(C)$ denote the group of automorphisms of $C$. Every $\sigma \in \operatorname{Aut}(C)$ induces an automorphism $\tilde{\sigma}$ of $V_{C}$ [Miyamoto 1996a, Section 5]. We call $\tilde{\sigma}$ a lift of $\sigma$. In particular, $\tilde{\sigma}$ acts as a permutation on $V^{\left(0^{n}\right)}$, that is,

$$
\tilde{\sigma}\left(v^{1} \otimes \cdots \otimes v^{n}\right)=v^{\sigma^{-1}(1)} \otimes \cdots \otimes v^{\sigma^{-1}(n)} \quad \text { for } v^{1} \otimes \cdots \otimes v^{n} \in V^{\left(0^{n}\right)} .
$$

Set

$$
t^{\alpha}:=\frac{1}{8} \sum_{i=1}^{8} e^{i}+\frac{1}{8} \sum_{\beta \in \widehat{\mathcal{H}}_{3}(4)}(-1)^{\mathrm{wt}(\beta * \alpha)} u^{\beta} \in V_{\widehat{\mathcal{H}}_{3}}
$$


for $\alpha \in \mathbb{F}_{2}^{8}$. It is known that $t^{\alpha}$ is an Ising vector of $\sigma$-type of $V_{\widehat{\mathcal{H}}_{3}}$; see [op. cit.]. Set $v_{i}:=\left(0^{i-1} 10^{n-i}\right) \in \mathbb{F}_{2}^{n}$.

Proposition 5.4 [Matsuo and Matsuo 2000, Proposition 2.4.1; Miyamoto 1999, Lemma 2.3]. The Hamming code VOA $V_{\widehat{\mathcal{H}}_{3}}$ has exactly three Ising frames:

$I_{0}:=\left\{e^{i} \mid 1 \leq i \leq 8\right\}, \quad I_{1}:=\left\{t^{\nu_{i}} \mid 1 \leq i \leq 8\right\}, \quad$ and $\quad I_{2}:=\left\{t^{\nu_{1}+v_{i}} \mid 1 \leq i \leq 8\right\}$.

Moreover, if $f \in I_{a}$, then $\sigma_{f}\left(I_{b}\right)=I_{c}$ if $\{a, b, c\}=\{0,1,2\}$, where $\sigma_{f}$ is the involution defined in (2-2).

Let $C$ be a binary code. Set

$$
\mathcal{D}(C):=\left\{D \subset C \mid D \cong \hat{\mathcal{H}}_{3} \text { and } \#(\operatorname{supp}(\alpha) \cap \operatorname{supp}(D)) \in 2 \mathbb{Z} \text { for all } \alpha \in C\right\},
$$

where

$$
\operatorname{supp}(D):=\bigcup_{d \in D} \operatorname{supp}(d)
$$

Let $I\left(V_{C}\right)$ denote the set of all Ising vectors of $\sigma$-type of $V_{C}$.

Proposition 5.5 [Lam et al. 2007, Proposition 3.8, Lemma 3.10]. Let $C$ be a binary code of length $n$ whose minimum weight is at least 3 , and $f \in I\left(V_{C}\right)$. If $f \notin\left\{e^{i}\right\}_{i=1}^{n}$, then there exists $D \in \mathcal{D}(C)$ such that $f \in V_{D} \subset V_{C}$ and $f$ is of the form (5-1) in $V_{D}$. Also, if $f \in V_{D} \subset V_{C}$ is an Ising vector of $\sigma$-type for $D \in \mathcal{D}(C)$, then $f \in I\left(V_{C}\right)$.

The following proposition for the VOA case has been obtained in [Lam et al. 2007, Proposition 3.13]. Using the same argument, one can also show the SVOA case.

Proposition 5.6. Let $C$ be a binary code whose minimum weight is at least 3. Then $\operatorname{Aut}\left(V_{C}\right)$ is generated by $\left\{\sigma_{f} \mid f \in I\left(V_{C}\right)\right\}$ and the lift of $\operatorname{Aut}(C)$.

Examples of code SVOAs of class $\mathcal{S}^{\mathbf{5}}$. The SVOA $L\left(\frac{1}{2}, 0\right) \oplus L\left(\frac{1}{2}, \frac{1}{2}\right)$ is clearly of class $\mathcal{S}^{\infty}$. Note that $V_{\mathcal{E}_{8}}$ is isomorphic to the lattice VOA $V_{D_{4}}$, and $V_{\widehat{\mathcal{H}}_{3}}$ and $V_{\widehat{\mathcal{H}}_{4}}$ are isomorphic to the lattice-type VOAs $V_{\sqrt{2} D_{4}}^{+}$and $V_{\sqrt{2} E_{8}}^{+}$, respectively; see [Dong et al. 1998; Lam et al. 2007]. It was shown in [Maruoka et al. 2016; Hashikawa and Shimakura 2016] that

$$
V_{D_{4}}, \quad V_{\sqrt{2} D_{4}}^{+}, \quad \text { and } \quad V_{\sqrt{2} E_{8}}^{+}
$$

are of class $\mathcal{S}^{5}$. Therefore the code VOAs are also of class $\mathcal{S}^{5}$. By Lemma 5.3(2), their minimal conformal weight spaces are conformal 5-designs. Hence we show that the remaining code SVOAs $V_{E\left(\mathcal{H}_{4}\right)}$ and $V_{\mathcal{H}_{4}}$ are also of class $\mathcal{S}^{5}$. 
Note that $\hat{\mathcal{H}}_{3}$ and $\mathcal{H}_{4}$ are generated by the rows of the following matrices.

$$
\widehat{\mathcal{H}}_{3}:\left[\begin{array}{ll}
0000 & 1111 \\
1111 & 0000 \\
0011 & 0011 \\
0101 & 0101
\end{array}\right] \text {, }
$$

$\mathcal{H}_{4}:\left[\begin{array}{llll}0001 & 0001 & 0001 & 000 \\ 0010 & 0010 & 0010 & 001 \\ 0100 & 0100 & 0100 & 010 \\ 1000 & 1000 & 1000 & 100 \\ 0101 & 0000 & 0101 & 000 \\ 1010 & 0000 & 1010 & 000 \\ 1100 & 0000 & 1100 & 000 \\ 0000 & 1111 & 0000 & 000 \\ 1111 & 0000 & 0000 & 000 \\ 0011 & 0011 & 0000 & 000 \\ 0101 & 0101 & 0000 & 000\end{array}\right]$.

It is easily seen from (5-2) that $\mathcal{D}\left(\mathcal{H}_{4}\right) \neq \varnothing$. Also, it is known that $\operatorname{Aut}\left(\mathcal{H}_{4}\right)$ acts doubly transitively on $\Omega_{15}$ [MacWilliams and Sloane 1977, Chapter 13, Theorem 9.24, and Problem (9)].

Theorem 5.7. The code SVOAs $V_{\mathcal{H}_{4}}$ and $V_{E\left(\mathcal{H}_{4}\right)}$ are of class $\mathcal{S}^{5}$.

Proof. Obviously, $V_{E\left(\mathcal{H}_{4}\right)}$ is the even part of $V_{\mathcal{H}_{4}}$. Now by Proposition 5.2 and Lemma 5.3(1), it is sufficient to show that

$$
\left(V_{\mathcal{H}_{4}}^{0}\right)_{5}^{\operatorname{Aut}\left(V_{\mathcal{H}_{4}}\right)}=\left(V_{\omega}\right)_{5} .
$$

A basis of $\left(V_{\omega}\right)_{5}$ is given by $\{L(-5) \mathbf{1}, L(-3) L(-2) \mathbf{1}\}$ because the central charge of $V_{\mathcal{H}_{4}}$ is neither 0 nor $-\frac{22}{5}$ (see Section 3.2). Note that for $n \in \mathbb{Z}$,

$$
L(n)=\sum_{i=1}^{15} L^{e^{i}}(n), \quad \text { where } L^{e^{i}}(n)=e_{(n+1)}^{i} \quad \text { for } 1 \leq i \leq 15 .
$$

Let $P$ be the subgroup of $\operatorname{Aut}\left(V_{C}\right)$ generated by $\left\{\sigma_{e^{i}} \mid 1 \leq i \leq 15\right\}$. Since $\sigma_{e^{i}}$ acts as $(-1)^{\mathrm{wt}\left(\alpha * v_{i}\right)}$ on $V^{\alpha}$ for $\alpha \in C$, the fixed point subspace of $P$ in $\left(V_{C}\right)_{5}$ is

$$
V_{5}^{\left(0^{15}\right)}=\left\langle L^{e^{i}}(-5) \mathbf{1}, L^{e^{i}}(-3) L^{e^{j}}(-2) \mathbf{1}|1 \leq i, j \leq 15|_{\mathbb{C}} .\right.
$$

Set $X:=\sum_{i=1}^{15} L^{e^{i}}(-3) L^{e^{i}}(-2) \mathbf{1}$. Then

$$
L(-3) L(-2) \mathbf{1}=X+\sum_{1 \leq i \neq j \leq 15} L^{e^{i}}(-3) L^{e^{j}}(-2) \mathbf{1} .
$$

The double transitivity of $\operatorname{Aut}\left(\mathcal{H}_{4}\right)$ gives

$$
\begin{aligned}
\left(V^{\left(0^{15}\right)}\right)_{5}^{\operatorname{Aut}\left(\mathcal{H}_{4}\right)} & =\left\langle L(-5) \mathbf{1}, X, \sum_{1 \leq i \neq j \leq 15} L^{e^{i}}(-3) L^{e^{j}}(-2) \mathbf{1}\right\rangle_{\mathbb{C}} \\
& =\langle L(-5) \mathbf{1}, L(-3) L(-2) \mathbf{1}, X\rangle_{\mathbb{C}} .
\end{aligned}
$$




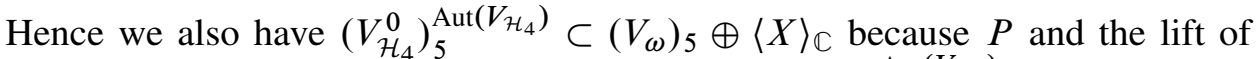
$\operatorname{Aut}\left(\mathcal{H}_{4}\right)$ are subgroups of $\operatorname{Aut}\left(V_{\mathcal{H}_{4}}\right)$. We show $X \notin\left(V_{\mathcal{H}_{4}}^{0}\right)_{5}^{\operatorname{Aut}\left(V_{\mathcal{H}_{4}}\right)}$. Let $D \in \mathcal{D}\left(\mathcal{H}_{4}\right)$ such that $\operatorname{supp}(D)=\{1, \ldots, 8\}$. By $(5-2)$, we can take such a subcode. Let $\left\{f^{i}\right\}_{i=1}^{8}$ and $\left\{g^{i}\right\}_{i=1}^{8}$ be distinct Ising frames of $V_{D}$ except for $\left\{e^{i}\right\}_{i=1}^{8}$ (see Proposition 5.4). We see from Proposition 5.5 that $f^{i}$ and $g^{i}$ are also Ising vectors of $\sigma$-type of $V_{\mathcal{H}_{4}}$. By Proposition 5.4,

$$
\sigma_{f^{1}}(X)=\sum_{i=1}^{8} L^{g^{i}}(-3) L^{g^{i}}(-2) \mathbf{1}+\sum_{i=9}^{15} L^{e^{i}}(-3) L^{e^{i}}(-2) \mathbf{1},
$$

where $L^{g^{i}}(n)=g_{(n+1)}^{i}$ for $1 \leq i \leq 8$ and $n \in \mathbb{Z}$. By direct computation, $\sigma_{f^{1}}(X) \neq X$. Therefore the assertion holds.

Remark 5.8. As already mentioned before, the cases of $V_{\widehat{\mathcal{H}}_{3}}$ and $V_{\widehat{\mathcal{H}}_{4}}$ have already been obtained in [Hashikawa and Shimakura 2016]. By using the same method as in Theorem 5.7, one can also show these cases.

In conclusion, we obtain the following.

Theorem 5.9. The code SVOAs associated to the codes in Theorem 4.8 are of class $\mathcal{S}^{5}$.

As a corollary of Theorems 4.8 and 5.9, the following holds.

Corollary 5.10. Let $C$ be a binary code. Then the minimal conformal weight space of $V_{C}$ is a conformal 4-design based on $V_{C}^{0}$ if and only if $V_{C}$ is of class $\mathcal{S}^{5}$.

Remark 5.11. It is known that the code VOA $V_{E\left(\mathcal{H}_{4}\right)}$ is isomorphic to the commutant subalgebra of an Ising vector in the VOA $V_{\sqrt{2} E_{8}}^{+}$[Lam et al. 2007, Section 4 and Corollary 5.6].

\section{Acknowledgments}

The author would like to thank Hiroki Shimakura for discussions and many valuable suggestions. He also thanks Hiroshi Yamauchi for helpful comments and Yuta Watanabe for discussions of coding theory and combinatorial design theory.

\section{References}

[Assmus and Key 1992] E. F. Assmus, Jr. and J. D. Key, Designs and their codes, Cambridge Tracts in Mathematics 103, Cambridge University Press, 1992. MR 1192126 Zbl 0762.05001

[Assmus and Mattson 1969] E. F. Assmus, Jr. and H. F. Mattson, Jr., "New 5-designs", J. Combinatorial Theory 6 (1969), 122-151. MR 0272647 Zbl 0179.02901

[Dong et al. 1994] C. Dong, G. Mason, and Y. Zhu, "Discrete series of the Virasoro algebra and the moonshine module", pp. 295-316 in Algebraic groups and their generalizations: quantum and infinite-dimensional methods (University Park, PA, 1991), edited by W. J. Haboush and B. J. 
Parshall, Proc. Sympos. Pure Math. 56, Amer. Math. Soc., Providence, RI, 1994. MR 1278737 Zbl 0813.17019

[Dong et al. 1998] C. Dong, R. L. Griess, Jr., and G. Höhn, "Framed vertex operator algebras, codes and the Moonshine module", Comm. Math. Phys. 193:2 (1998), 407-448. MR 1618135 Zbl 0908.17018

[Frenkel et al. 1993] I. B. Frenkel, Y.-Z. Huang, and J. Lepowsky, On axiomatic approaches to vertex operator algebras and modules, vol. 104, Mem. Amer. Math. Soc. 494, 1993. MR 1142494 Zbl 0789.17022

[Hashikawa and Shimakura 2016] T. Hashikawa and H. Shimakura, "Classification of the vertex operator algebras $V_{L}^{+}$of class $\mathcal{S}^{4}$,, J. Algebra 456 (2016), 151-181. MR 3484139 Zbl 1335.17015

[Höhn 2008] G. Höhn, "Conformal designs based on vertex operator algebras", Adv. Math. 217:5 (2008), 2301-2335. MR 2388095 Zbl 1157.17008

[Höhn et al. 2012] G. Höhn, C. H. Lam, and H. Yamauchi, "McKay's $E_{7}$ observation on the Baby Monster”, Int. Math. Res. Not. 2012:1 (2012), 166-212. MR 2874931 Zbl 1267.17033

[Kac 1998] V. Kac, Vertex algebras for beginners, 2nd ed., University Lecture Series 10, American Mathematical Society, Providence, RI, 1998. MR 1651389 Zbl 0924.17023

[Kac and Raina 1987] V. G. Kac and A. K. Raina, Bombay lectures on highest weight representations of infinite-dimensional Lie algebras, Adv. Ser. Math. Phys. 2, World Scientific, Teaneck, NJ, 1987. MR 1021978 Zbl 0668.17012

[Lam et al. 2007] C. H. Lam, S. Sakuma, and H. Yamauchi, "Ising vectors and automorphism groups of commutant subalgebras related to root systems", Math. Z. 255:3 (2007), 597-626. MR 2270290 Zbl 1139.17010

[Li 1994] H. S. Li, "Symmetric invariant bilinear forms on vertex operator algebras", J. Pure Appl. Algebra 96:3 (1994), 279-297. MR 1303287 Zbl 0813.17020

[MacWilliams and Sloane 1977] F. J. MacWilliams and N. J. A. Sloane, The theory of error-correcting codes, I, North-Holland, Amsterdam-New York-Oxford, 1977. MR 0465509 Zbl 0369.94008

[Maruoka et al. 2016] H. Maruoka, A. Matsuo, and H. Shimakura, "Classification of vertex operator algebras of class $\mathcal{S}^{4}$ with minimal conformal weight one", preprint, 2016. arXiv math.QA/1509.05529

[Matsuo 2001] A. Matsuo, "Norton's trace formulae for the Griess algebra of a vertex operator algebra with larger symmetry", Comm. Math. Phys. 224:3 (2001), 565-591. MR 1871901

[Matsuo and Matsuo 2000] A. Matsuo and M. Matsuo, "The automorphism group of the Hamming code vertex operator algebra”, J. Algebra 228:1 (2000), 204-226. MR 1760962 Zbl 0978.17023

[Miyamoto 1996a] M. Miyamoto, "Binary codes and vertex operator (super)algebras", J. Algebra 181:1 (1996), 207-222. MR 1382033 Zbl 0857.17026

[Miyamoto 1996b] M. Miyamoto, "Griess algebras and conformal vectors in vertex operator algebras", J. Algebra 179:2 (1996), 523-548. MR 1367861 Zbl 0964.17021

[Miyamoto 1999] M. Miyamoto, "A Hamming code vertex operator algebra and construction of vertex operator algebras”, J. Algebra 215:2 (1999), 509-530. MR 1686204 Zbl 0929.17035

[Miyamoto 2004] M. Miyamoto, "A new construction of the Moonshine vertex operator algebra over the real number field", Ann. of Math. (2) 159:2 (2004), 535-596. MR 2081435 Zbl 1133.17017

[Tuite 2009] M. P. Tuite, "Exceptional vertex operator algebras and the Virasoro algebra", pp. 213225 in Vertex operator algebras and related areas (Normal, IL, 2008), edited by M. Bergvelt et al., Contemp. Math. 497, Amer. Math. Soc., Providence, RI, 2009. MR 2568410 Zbl 1225.17034

[Tuite and Van 2014] M. P. Tuite and H. D. Van, "On exceptional vertex operator (super)algebras", pp. 351-384 in Developments and retrospectives in Lie theory, edited by G. Mason et al., Dev. Math. 38, Springer, 2014. MR 3308791 Zbl 06463592 
[Venkov 2001] B. Venkov, "Réseaux et designs sphériques", pp. 10-86 in Réseaux euclidiens, designs sphériques et formes modulaires, edited by J. Martinet, Monogr. Enseign. Math. 37, Enseignement Math., Geneva, 2001. MR 1878745 Zbl 1139.11320

[Yamauchi 2004] H. Yamauchi, A theory of simple current extensions of vertex operator algebras and applications to the Moonshine vertex operator algebra, Ph.D. thesis, University of Tsukuba, 2004, available at http://www.math.twcu.ac.jp/ yamauchi/math/phd/myphd.pdf.

[Yamauchi 2005] H. Yamauchi, "2A-orbifold construction and the baby-monster vertex operator superalgebra”, J. Algebra 284:2 (2005), 645-668. MR 2114573 Zbl 1147.17314

[Yamauchi 2014] H. Yamauchi, "Extended Griess algebras and Matsuo-Norton trace formulae", pp. 75-107 in Conformal field theory, automorphic forms and related topics (Heidelberg, Germany, 2011), edited by W. Kohnen and R. Weissauer, Springer, Berlin, 2014. Zbl 06489870

Received November 15, 2015.

TOMONORI HASHIKAWA

TOHOKU UNIVERSITY

\#113 49-154

AobA-Ku SENDAI-SHI MiYAgI-KEN

SENDAI 980-0866

JAPAN

t.hashikawa@ims.is.tohoku.ac.jp 


\title{
PACIFIC JOURNAL OF MATHEMATICS
}

Founded in 1951 by E. F. Beckenbach (1906-1982) and F. Wolf (1904-1989)

$$
\text { msp.org/pjm }
$$

\section{EDITORS}

\author{
Don Blasius (Managing Editor) \\ Department of Mathematics \\ University of California \\ Los Angeles, CA 90095-1555 \\ blasius@math.ucla.edu
}

\author{
Paul Balmer \\ Department of Mathematics \\ University of California \\ Los Angeles, CA 90095-1555 \\ balmer@math.ucla.edu \\ Robert Finn \\ Department of Mathematics \\ Stanford University \\ Stanford, CA 94305-2125 \\ finn@math.stanford.edu \\ Sorin Popa \\ Department of Mathematics \\ University of California \\ Los Angeles, CA 90095-1555 \\ popa@math.ucla.edu
}

\author{
Vyjayanthi Chari \\ Department of Mathematics \\ University of California \\ Riverside, CA 92521-0135 \\ chari@math.ucr.edu \\ Kefeng Liu \\ Department of Mathematics \\ University of California \\ Los Angeles, CA 90095-1555 \\ liu@math.ucla.edu \\ Igor Pak \\ Department of Mathematics \\ University of California \\ Los Angeles, CA 90095-1555 \\ pak.pjm@gmail.com \\ Paul Yang \\ Department of Mathematics \\ Princeton University \\ Princeton NJ 08544-1000 \\ yang@math.princeton.edu
}

\section{PRODUCTION}

Silvio Levy, Scientific Editor, production@msp.org

\section{SUPPORTING INSTITUTIONS}

ACADEMIA SINICA, TAIPEI

CALIFORNIA INST. OF TECHNOLOGY

STANFORD UNIVERSITY

UNIV. OF BRITISH COLUMBIA

UNIV. OF CALIFORNIA, BERKELEY

UNIV. OF CALIFORNIA, DAVIS

UNIV. OF CALIFORNIA, LOS ANGELES

UNIV. OF CALIFORNIA, RIVERSIDE

UNIV. OF CALIFORNIA, SAN DIEGO

UNIV. OF CALIF., SANTA BARBARA
KEIO UNIVERSITY

MATH. SCIENCES RESEARCH INSTITUTE

NEW MEXICO STATE UNIV.

OREGON STATE UNIV.
Daryl Cooper

Department of Mathematics

University of California

Santa Barbara, CA 93106-3080 cooper@math.ucsb.edu

Jiang-Hua Lu

Department of Mathematics

The University of Hong Kong

Pokfulam Rd., Hong Kong

jhlu@maths.hku.hk

$$
\text { Jie Qing }
$$

Department of Mathematics

University of California

Santa Cruz, CA 95064

qing@ cats.ucsc.edu

\author{
UNIV. OF CALIF., SANTA CRUZ \\ UNIV. OF MONTANA \\ UNIV. OF OREGON \\ UNIV. OF SOUTHERN CALIFORNIA \\ UNIV. OF UTAH \\ UNIV. OF WASHINGTON \\ WASHINGTON STATE UNIVERSITY
}

These supporting institutions contribute to the cost of publication of this Journal, but they are not owners or publishers and have no responsibility for its contents or policies.

See inside back cover or msp.org/pjm for submission instructions.

The subscription price for 2016 is US $\$ 440 /$ year for the electronic version, and \$600/year for print and electronic.

Subscriptions, requests for back issues and changes of subscriber address should be sent to Pacific Journal of Mathematics, P.O. Box 4163, Berkeley, CA 94704-0163, U.S.A. The Pacific Journal of Mathematics is indexed by Mathematical Reviews, Zentralblatt MATH, PASCAL CNRS Index, Referativnyi Zhurnal, Current Mathematical Publications and Web of Knowledge (Science Citation Index).

The Pacific Journal of Mathematics (ISSN 0030-8730) at the University of California, c/o Department of Mathematics, 798 Evans Hall \#3840, Berkeley, CA 94720-3840, is published twelve times a year. Periodical rate postage paid at Berkeley, CA 94704, and additional mailing offices. POSTMASTER: send address changes to Pacific Journal of Mathematics, P.O. Box 4163, Berkeley, CA 94704-0163.

PJM peer review and production are managed by EditFLOW ${ }^{\circledR}$ from Mathematical Sciences Publishers.

PUBLISHED BY

\section{I. mathematical sciences publishers}

nonprofit scientific publishing

http://msp.org/

(C) 2016 Mathematical Sciences Publishers 


\section{PACIFIC JOURNAL OF MATHEMATICS}

Volume $284 \quad$ No. $1 \quad$ September 2016

Bitwist manifolds and two-bridge knots

JAmes W. CANNON, William J. Floyd, LEeR LAMbert,

WALTER R. PARry and Jessica S. PurCELL

Recognizing right-angled Coxeter groups using involutions

Charles Cunningham, Andy Eisenberg, Adam Piggott and KIM RUANE

On Yamabe-type problems on Riemannian manifolds with boundary

Marco Ghimenti, Anna Maria Micheletti and Angela

PISTOIA

Quantifying separability in virtually special groups

MARK F. HAGEN and PRIYAM PATEL

Conformal designs and minimal conformal weight spaces of vertex operator superalgebras

TOMONORI HASHIKAWA

Coaction functors

S. KALiszewski, Magnus B. LANDSTAD and John QuigG

Cohomology and extensions of braces

VICTORIA LEBED and LEANDRO VENDRAMIN

Noncommutative differentials on Poisson-Lie groups and pre-Lie algebras

SHAHN MAJID and WEN-QING TAO 